18 3. Present address: Biomedical Image Analysis Laboratory. Oxford Institute of 19 Biomedical Engineering, Department of Engineering Science- University of Oxford. Old 20 Road Campus Research Building. Headington, Oxford OX3 7DQ, UK

21

22

29 Running tittle: PERK couples ER and microtubule dynamics

31 Word count: 4559 words

\section{PERK-dependent reciprocal crosstalk between ER and non-centrosomal microtubules coordinates ER architecture and cell shape}

\author{
Miguel Sánchez-Álvarez, $\mathrm{PhD}^{1,2} \S$; Fidel Lolo, $\mathrm{PhD}^{2}$; Heba Sailem, $\mathrm{PhD}^{1,3}$; \\ Patricia Pascual-Vargas, $\mathrm{PhD}^{1,4}$; Giulio Fulgoni, $\mathrm{MSc}^{2}$; Mar Arias-García, MSc ${ }^{1}$;
} Miguel Ángel del Pozo, MD, $\mathrm{PhD}^{2}$; Chris Bakal, $\mathrm{PhD}^{1} \S$ Mechanoadaptation and Caveolae Biology laboratory. Area of Cell and Developmental Biology. National Centre for Cardiovascular Research (CNIC). c/Melchor Fernandez Almagro, 3. CP 28029, Madrid, Spain.

(1)

4. Present address: Department of Cell and Developmental Biology, University College London. Gower Street, London WC1E 6BT, UK. (1)

$\S$ To whom correspondence should be addressed: msancheza@cnic.es, cbakal@icr.ac.uk (1) (1) (1) 


\section{ABSTRACT:}

35 The architecture of the endoplasmic reticulum (ER) is tightly controlled as a key 36 determinant of its function. Its dynamics are linked to those of the cytoskeleton, but our 37 understanding of how this coordination occurs and what its functional relevance is, is 38 limited. We found the Unfolded Protein Response (UPR) transducer EIF2AK3/PERK is 39 essential for acute stress-induced peripheral redistribution and remodeling of the ER, 40 through elF2a phosphorylation and translation initiation shutdown. PERK-mediated elF2a 41 phosphorylation can be bypassed by blocking ribosome activity; by depleting microtubule42 anchoring ER proteins such as REEP4, p180/RRBP1 and Climp63/CKAP4; or by 43 disrupting the microtubule cytoskeleton. Notably, specific disruption of non-centrosomal 44 microtubules, but not centrosome depletion, relieved blockade of ER redistribution in 45 PERK-deficient cells. Conversely, PERK deficiency stabilized non-centrosomal 46 microtubules, promoting polarized protrusiveness in epithelial cells and neuroblasts. We 47 propose that PERK coordinates ER architecture and homeostasis with cell 48 morphogenesis by coupling ER remodeling and non-centrosomal MT dynamics. 


\section{INTRODUCTION:}

56 The eukaryotic endoplasmic reticulum (ER) is an intricate system of intracellular 57 membrane domains delimiting a single luminal space, continuous with the outer nuclear 58 envelope. The architecture of the ER and its dynamics contribute to the several essential 59 functions of this organelle, including calcium and redox homeostasis, complex lipid 60 metabolism, management of other endomembrane systems, and the maturation and 61 assisted folding of $\sim 30 \%$ of the proteome $e^{1}$. ER membrane subdomains can adopt discrete 62 shapes (including ER 'tubules' (peripheral, reticular tubes of ER, with rather low densities 63 of associated ribosomes) and ER 'sheets' (flat enlargements or "cisterns" of peripheral $64 \mathrm{ER}$, usually rich in bound polysomes) ${ }^{2}$. The local concentration and activity of "ER 65 shapers" such as reticulons, atlastins and other auxiliary resident proteins ${ }^{3}$ determines the 66 shape of the ER. Accordingly, physical expansion of the ER is an integral component of its 67 adaptive response to functional imbalances (generically termed "ER stress"). ER 68 expansion is integrated with the Unfolded Protein Response of the ER (UPR ${ }^{\mathrm{ER}}$ ), a 69 surveillance mechanism that continuously gauges ER luminal environment and membrane 70 integrity and engages adaptive programs as required ${ }^{5-7}$.

71 The UPR ${ }^{\mathrm{ER}}$ of higher eukaryotes comprises three main branches, each of them driven by 72 a specific, ER-resident transducer. Inositol Requiring enzyme 1 (IRE1; also known as 73 Endoplasmic Reticulum To Nucleus Signaling 1, ERN1) orchestrates a complex adaptive 74 transcriptional response through the unconventional splicing of XBP1 (X-box binding 75 protein 1) mRNA, eliciting its translation as a potent transcriptional transactivator of ER 76 chaperones and lipid anabolism ${ }^{7,8}$. Upon activated intramembrane cleavage, Activation 77 Transcription Factor 6 (ATF6) also promotes adaptive transcriptional programs, driving $78 \mathrm{red} / \mathrm{ox}$ regulation, ER chaperone expression and lipid metabolism enzymes ${ }^{7,9}$. Both UPR 79 branches are involved in ER membrane de novo synthesis and ER physical expansion ${ }^{10-}$ $80{ }^{12}$. The third branch is operated by the EIF2A kinase 3/PKR-like ER Kinase 81 (EIF2AK3/PERK), one of the four known stress-associated kinases in metazoans capable 82 of phosphorylating the essential translation regulator eukaryotic initiation factor $2 a$ (elF2 $\alpha$ ). 83 elF2 $\alpha$ is a GTPase subunit of the translation initiation ternary complex capped mRNA84 ribosomal subunit $60 \mathrm{~S}^{-e} \mathrm{eF}^{7}$; phosphorylation on its conserved Ser51 residue locks elF2 $\alpha$ 85 in its GDP-bound, inactive state and leads to sequestration of the ternary complex from 86 further assembling a processive ribosome. Therefore, elF2 $\alpha$ phosphorylation is a node 87 onto which several stress responses (including UPR through PERK) converge to regulate 88 protein synthesis. Limiting client protein overload in the stressed ER through translation 89 downregulation is thus commonly proposed as the main role of $\mathrm{PERK}^{7,13}$. However, 90 PERK-dependent protein translation attenuation is integrated with other functional outputs 
91 beyond control of client protein burden on the ER, such as regulation of calcium trafficking

92 and apoptosis ${ }^{14-16}$.

The ER communicates with other membrane-bound organelles, plasma

94 membrane subdomains, and the cytoskeleton ${ }^{17}$. While lower eukaryotes and plants mostly

95 rely on actin cytoskeleton dynamics for the shaping and partition of their $\mathrm{ER}^{18,19}$, the

96 metazoan ER is tightly coupled to the microtubule (MT) cytoskeleton ${ }^{20-23}$. Several integral

97 proteins of the ER membrane such as Stromal interaction molecule 1 (STIM1), Ribosome-

98 binding protein 1 (RRBP1/p180), cytoskeleton-linking membrane protein 63

99 (Climp63/CKAP4), Receptor expression-enhancing proteins 1-4 (REEPs1-4) or reticulons

100 can engage in physical contacts with $\mathrm{MTs}^{24-28}$. Extension, fusion and reticulation of ER

101 tubules are guided by dynamic microtubule bundles ${ }^{17}$. ER sheets also establish anchoring

102 interactions with microtubules, and they appear to do so preferentially through

103 p180/RRBP1 and Climp63, which in turn also establish interactions with

104 polysome/translocon complexes enriched in these areas of "rough" ER ${ }^{29,30}$. Importantly,

105 these interactions are a means by which changes in ER morphology can affect MT

106 organization, and viceversa. For example, p180/RRBP1 overexpression can induce

107 hyperstabilization of microtubule bundles and promote the formation of tight, collapsed

108 structures ${ }^{25}$.

109 We developed automated image-based RNAi screening procedures to explore

110 the genetic regulation of ER expansion and redistribution upon pharmacologically

111 challenging ER homeostasis. We found the UPR effector PERK is essential for ER

112 redistribution to the cell periphery during ER expansion upon induction of ER stress. This

113 activity is dependent on translation initiation shutdown through elF2 $\alpha$ phosphorylation, and

114 can be bypassed by blocking translation initiation and polysome disassembly, but not by

115 shutting down translation elongation. Combinatorial siRNA screening revealed that

116 depletion of proteins linking microtubules with the ER, such as REEP4, p180/RRBP1 and

117 Climp63/CKAP4, specifically rescued the ER collapse associated with disruption of PERK

118 signaling. Importantly, while centrosome depletion did not have an observable impact on

119 ER architecture dynamics, inhibition of CAMSAP2-stabilized non-centrosomal

120 microtubules promoted the peripheral expansion of ER sheet structures, and fully rescued

121 ER redistribution in PERK-depleted cells. Conversely, abrogation of PERK activity led to

122 phenotypes of increased polarity and low number of large protrusions in epithelial cells, for

123 which epistasis with non-centrosomal microtubules was also observed. Thus, during ER

124 stress PERK inhibits translation initiation at the ribosomes, which alters the coupling of ER

125 to non-centrosomal MTs through specific ER-MT linkers such as CKAP4/Climp63 and

126 RRBP1/p180, and both facilitates expansion and modulates MT cytoskeleton 
127 arrangement. Our observations highlight the existence of additional key roles for PERK on 128 cell homeostasis beyond the curbing of ER client protein synthesis. 


\section{RESULTS:}

\section{PERK is a novel regulator of ER redistribution during acute ER stress in epithelial}

132 cells

133 With the aim of exploring mechanisms determining ER architecture during ER stress, we

134 developed an image analysis pipeline for automated high-content ER morphology analysis

135 in single cells. (fig. $1 \mathrm{~A} ;{ }^{31,32}$ ). An array of features (periphery/perinuclear averaged intensity

136 ratio of the ER; image textures, reflecting different aspects of ER architecture; and

137 subcellular distribution) is extracted from single cells, together with other morphological

138 features of the whole cell (fig. $1 \mathrm{~A}$ and fig. S1A). To assess the validity of our approach to

139 capture significant changes in ER morphogenesis across different conditions, we first

140 compared wild type cells with cells exposed to the $\mathrm{N}$-glycosylation inhibitor tunicamycin,

141 which provokes acute ER stress and a prominent redistribution and expansion of the ER

142 in epithelial cells, together with changes in image texture (fig. 1A). The ratio of ER signal

143 density on the cell periphery as compared with the perinuclear region was informative of

144 adaption to ER stress (fig. 1A, right panels). We further tested this system by

145 interrogating, both in untreated and tunicamycin-treated cells, a small collection of

146 siRNAs, targeting well-established direct regulators of ER morphogenesis and

147 homeostasis (see Table S1). Notably, knockdown of Inositol Requiring Enzyme 1 alpha

148 (IRE1a), a key regulator of ER membrane expansion ${ }^{4,33}$, led to reduced peripheral ER

149 distribution across conditions (fig. 1B and S1B). Thus, we conclude we are able to

150 quantify physiologically relevant changes in ER morphology in single cells during ER

151 stress.

153 We found that depletion of the protein kinase $R(P K R)$-like endoplasmic reticulum kinase

154 (PERK/EIF2AK3), a well-established essential factor for ER homeostasis ${ }^{13}$, led to a

155 marked impairment for ER subcellular redistribution upon exposure to tunicamycin (fig.

156 1B-D and fig. S1A). Superresolution light microscopy and electron microscopy confirmed

157 that the ER of cells depleted of PERK is not rearranged to increase peripheral sheets in

158 response to ER stress, and collapses at the perinuclear region (fig. $1 \mathrm{E}$ and $\mathrm{F}$ ). To assess

159 that these phenotypes were not due to off-target effects, four different siRNA sequences

160 were chosen for secondary validation. qRT-PCR assays consistently showed a

161 downregulation of at least $\sim 80 \%$ in PERK mRNA levels upon transfection of any of the

162 tested siRNA sequences (fig. S1C). Transfection of all four siRNAs recapitulated the 163 phenotype of impaired peripheral ER redistribution upon tunicamycin challenge (fig. 2A,

164 lower row; fig. 2B). PERK knock-down in other cell lines of different origins, such as a 
165 transformed MCF10A clone (ATI clone, expressing the constitutively active H-Ras G12V 166 mutant) or two other tumour epithelial cell lines (HeLa and MDA-MB231) resulted in 167 identical phenotypes (fig. S1D and E). Furthermore, the phenotype of impaired ER 168 subcellular redistribution upon acute exposure to tunicamycin could also be followed 169 through live imaging of a stable MCF10A-derived cell line expressing EGFP-tagged 170 Sec61ß (fig. S1F).

172 We further validated our observations using the allosteric PERK kinase inhibitor 173 GSK2606414 (fig. 2C) for different times, previous to tunicamycin challenge. PERK kinase 174 inhibition again recapitulated the ER collapse associated with PERK siRNA depletion (fig. 175 2D and E). Of note, disruption of PERK kinase activity using this chemical inhibitor in 176 unchallenged cells also led to moderate phenotypic alterations regarding cell elongation 177 and peripheral ER architecture, similar to those observed for PERK siRNA-mediated 178 knock-down. PERK siRNA transfection or exposure to PERK kinase inhibitor were also 179 associated with ER collapse when cells were challenged with a different source of ER 180 stress, such as the sarco/endoplasmic reticulum Ca2+-ATPase inhibitor thapsigargin (fig. $1812 \mathrm{~F}$ and $\mathrm{G})$.

183 We further tested two established cell models, derived from the MCF10A epithelial line, 184 which reprogram PERK regulation and output. First, we looked into the phenotype of the 185 PERK- $\triangle \mathrm{C}$ cell line-which expresses a C-terminal truncated mutant with dominant 186 negative properties ${ }^{34,35}$ )_across different conditions. The PERK- $\Delta$ C-expressing cell line 187 exhibited partially impaired redistribution of their ER upon tunicamycin exposure (fig $2 \mathrm{H}$ 188 and J). We studied the behavior of another MCF10A-derived cell line, Fv2E-PERK, which 189 expresses a synthetic PERK construct by which it is possible to uncouple PERK activation 190 from canonical ER stress $\left({ }^{34,35}\right.$; fig. S2A). Exposure to the B/B homodimerizer AP20187 191 provoked apparent ER peripheral expansion in FV2E-PERK cells, but not in wild-type 192 MCF10A cells (fig. $2 \mathrm{I}$ and K). These observations support that the phenotypes we observe 193 are specifically derived from altered PERK kinase activity.

195 PERK/EIF2AK3 kinase role in remodeling of peripheral ER architecture depends on 196 elF2 $\alpha$ phosphorylation-driven translation attenuation

197 In certain cell models, PERK is required for the activation of the lipid anabolism 198 regulator Sterol Regulatory Element Binding Protein 1 (SREBP1; ${ }^{36}$ ). Thus we considered 
199 deficiency in ER expansion in PERK-deficient cells could be due to impaired lipid 200 anabolism or de novo membrane synthesis. However, in our hands, siRNA-mediated 201 depletion of PERK did not impact significantly on the activation of the canonical ER lipid 202 anabolism regulators such as SREBP1, or X-box binding protein 1 mRNA processing 203 (XBP1) ( ${ }^{10-12,36}$, fig. S2B). Furthermore, cells depleted for these regulators exhibited ER 204 phenotypes different from those displayed by cells knocked down for PERK (see above 205 Fig. S1B). Importantly, the increase in total ER membrane content upon ER stress 206 induction in PERK-depleted cells is comparable to that of wild-type cells (fig. S2C). Thus, 207 our observations suggested a potential novel role for PERK in the control of ER 208 architecture remodelling and subcellular redistribution during ER stress, distinct from de 209 novo ER membrane synthesis.

210 We next investigated the role of Activated Transcription Factor 4 (ATF4) in the 211 role of PERK-mediated ER expansion. ATF4 is a a key UPR transcriptional regulator 212 whose translation is dependent on the ribosomal frameshift elicited by PERK-dependent 213 elF2 $\alpha$ phosphorylation ${ }^{15}$. PERK depletion in our cell system blunted ER stress-driven 214 ATF4 translation (fig. S2D). But siRNA-mediated depletion of ATF4 did not recapitulate 215 the phenotypic characteristics associated with the abrogation of PERK activity, and in fact 216 led to moderate but significantly opposite effects, regarding ER remodeling, cell spreading 217 and overall morphology across the tested conditions (fig. S2E and F). These observations 218 support the notion that the role of PERK in the phenotypes we observe is not dependent 219 on downstream gene expression programs driven by the ATF4/CHOP axis.

We hypothesized that PERK-dependent translation of targets in addition to ATF4

221 might directly underlie stress-induced ER redistribution. We thus implemented a 222 multiplexed method to quantify global translation activity on a single-cell basis while 223 simultaneously determining the subcellular distribution and architecture of the ER. 224 Polysomes engaged in active translation are immunolabelled and visualized after a brief 225 pulse of puromycin (puromycylation; hereon termed 'PMY' (fig. $S 3 A,{ }^{37}$ ). The signal is 226 highly specific because either omission of PMY, or robust disruption of ribosomal 227 assembly through different approaches before exposure to a puromycin pulse, completely 228 abolish immunolabelling (fig. S3B). Acute induction of ER stress leads to a substantial 229 decrease in PMY signal, which correlates with upregulation of phosphor- elF2 $\alpha$ signal, in a 230 PERK-dependent manner (fig. 3A). We plotted ER redistribution measurements against 231 PMY signal across different conditions for single wild-type MCF10A cells (fig. 3B). 232 Importantly, translation activity (as judged by PMY intensity) and degree of relative 233 peripheral ER density exhibited an inverse correlation on a single-cell basis. Moreover, 234 phosphor-elF2 $\alpha$ signal (inhibition of translation) followed an opposite (positive) correlation 
235 pattern with ER expansion on a single-cell basis (fig. 3B, lower panels). Thus as expected

236 acute induction of ER stress robustly increased ER expansion but suppressed translation

237 globally (fig. 3B, upper panels).

Importantly, knockdown of PERK blunted these responses (fig. 3C). Detailed

240 confocal analysis showed that peripheral expanded ER structures in cells subject to ER

241 stress showed reduced overlap with PMY signal, supporting that translational shutdown is

242 a required step for ER adaptive remodeling (fig. 3D). Of note, induction of Fv2E-PERK

243 oligomerization was associated with significant increases in the relative expansion of the

244 ER, which correlated with elF2alpha phosphorylation and translation attenuation levels, in

245 the absence of ER stress (fig. S3C). Thus ER expansion during stress correlates with

246 reduced polysome assembly and suppression of translation.

247 We next tested whether bypassing PERK-dependent shutdown of translation

248 initiation using different inhibitors of protein synthesis, could rescue the suppression of ER

249 expansion associated with PERK depletion/inhibition during ER stress challenge. Brief

250 exposure of tunicamycin-challenged cells to translation initiation inhibition (harringtonin or

251 puromycin) completely reverted the ER collapse specifically associated with abolition of

252 PERK function (fig. 3E). Interestingly, the rescue was associated rather specifically with

253 blockade of translation initiation and polysome assembly, because exposure to

254 cycloheximide, a drug intervening the elongation step of protein translation and stabilizing

255 polysomes in short term timeframes, was unable to revert the PERK siRNA phenotype

256 (fig. 3E), even under conditions that profoundly inhibit de novo protein synthesis ${ }^{29}$. Our

257 observations support a working model whereby deficient regulation of translation initiation

258 per se, and not potential client protein overload, is the cause leading to blunted ER

259 redistribution in PERK-deficient cells during ER stress.

260 We performed a set of experiments to elucidate the requirement for elF2 $\alpha$ we

261 had observed upon disruption of PERK activity. Cotransfection of an elF2 $\alpha$ S51D

262 phospho-mimetic mutant, but not of a wild type construct, rescued the ER collapse

263 observed in PERK-depleted, tunicamycin-challenged cells (fig. 4A-C). Notably, we

264 observed moderate but significant increases of ER relative expansion in the absence of

265 ER stress challenge both in wild-type and PERK-depleted cells upon ectopic expression

266 of the phospho-mimicking mutant (fig. 4B and C), supporting a model whereby active

267 mRNA translation per se is the main factor dictating relative ER distribution and

268 architecture in our experimental model. 
270 expansion during ER stress, we performed experiments where we stimulated the activity

271 of an alternative elF2 $\alpha$ kinase during ER stress in PERK-deficient cells. Sodium arsenite

272 specifically stimulates elF2 $\alpha$ phosphorylation through HRI/EIF2AK1 (fig. 4D). Of note,

273 exposure to sodium arsenite led to ER expansion in the absence of ER-targeted drugs,

274 both in wild type cells and PERK-depleted cells (fig. 4E and F).Moreover, sodium arsenite

275 rescued the ER collapse phenotype associated with PERK depletion/inhibition (fig. 4E and

$276 \mathrm{~F}$ ). In accordance with the idea that ER expansion is dependent on elF2 $\alpha$ phosphorylation

277 status, depletion of the HRI kinase prevented sodium arsenite-derived rescue (fig 4E and

278 F). Finally, we tested the effect of artificially delaying the dynamics of elF2alpha

279 phosphorylation using a specific inhibitor of the PPP1R15B phosphatase, guanabenz ${ }^{38}$;

280 which blocks elF2alpha dephosphorylation upon clearance of ER stress (fig. 4G).

281 Consistent with an intrinsic role for elF2alpha-dependent regulation of translational activity

282 in the cell to determine ER subcellular distribution, exposure to guanabenz delayed the

283 reverse redistribution of the ER associated with ER stress clearance in wild type MCF10A

284 cells (fig. 4G-I). Taken together, these observations support that the inhibition of

285 translation initiation and polysome assembly through elF2alpha phosphorylation

286 underpins PERK-mediated ER expansion.

288 The integrity of non-centrosomal MTs determines PERK-dependent regulation of ER

289 architecture

290 We next decided to look for additional players in this translation activity-dependent

291 regulation of ER architecture. Using a double siRNA screening approach, we queried a

292 focused list of structural ER "shapers", known to play specific roles in ER subdomain

293 definition (fig. 5A) to assess whether their inhibition suppressed or enhanced the ER

294 morphogenesis defects observed upon PERK depletion. siRNAs targeting these proteins

295 were effective and did not affect the levels of PERK mRNA (fig. S4A). This small siRNA

296 library was either transfected alone or cotransfected with PERK-targeting siRNA. Finally,

297 these combinations and their corresponding control and PERK siRNA-only controls were

298 exposed to either tunicamycin or vehicle (DMSO) alone. Depletion of REEP4, 299 p180/RRBP1 and Climp63/CKAP4 rescued the PERK siRNA phenotype of ER 'collapse'

300 upon tunicamycin exposure (fig. 5B; highlighted). But this rescue did not correlate with an

301 alteration of protein translation, as inferred from PMY staining (fig. S4B). Curiously, these

302 hits are mostly conserved in higher metazoans, and orthologs are not found across clades

303 where EIF2AK3/PERK is absent ${ }^{39}$, suggesting a co-evolved functional relationship (fig. 
304 S4C). We conclude ER collapse in PERK-deficient cells involves an altered regulation of 305 specific ER shapers.

306 Because REEP4, p180/RRBP1 and Climp63/CKAP4 are involved in the linkage between 307 the ER and the microtubule (MT) network ${ }^{25,28,40}$, we tested the involvement of MT stability 308 and organization in the phenotypes associated with PERK deficiency. Disruption of MT 309 polymerization upon exposure to nocodazole had a profound impact on ER architecture, 310 promoting a relative redistribution and an increase in sheet-like domains in untreated wild311 type cells (fig. 6A). Nocodazole treatment also completely reverted the observed ER 312 collapse associated with PERK depletion (fig. 6A). Importantly, this effect is not 313 dependent on translation activity per se, because nocodazole-treated cells are still 314 competent in engagement of ribosomes in protein synthesis at the times and 315 concentrations tested (fig. S5A). Thus, we conclude ER collapse in the absence of PERK 316 activity is driven by interactions between ER shapers and MTs.

317 We wondered whether specific MT populations might be involved in linker morphogenesis. 318 Two major classes of microtubule populations exist: microtubules nucleated at 319 centrosomes, and non-centrosomal microtubule bundles ${ }^{41}$. We first assessed whether 320 centrosomal microtubules were required to determine ER cell distribution and architecture 321 by depleting centrosome structures from MCF10A cells using the polo-like kinase 4 (PLK4) 322 inhibitor centrinone ${ }^{42}$. The ER of cells devoid of centrosomal MTs were phenotypically 323 similar to those in PERK/EIF2AK3 knock-down cells, indicating that depletion of MTs 324 nucleated at the centrosome does not rescue the ER expansion defects associated with 325 PERK inhibition (fig. S5B). Next, we depleted mRNAs encoding the calmodulin regulated 326 spectrin associated protein family member 2 (CAMSAP2), a well-established minus-end 327 stabilizer of non-centrosomal $\mathrm{MTs}^{41,43}$ (fig. 6B). Cells deficient for stabilization of non328 centrosomal MTs alone exhibited a moderate phenotype of increased peripheral ER 329 cistern-like structures (fig. 6C and D). Importantly, CAMSAP2-deficient cells simultaneously 330 knocked down for PERK rescued the collapsed perinuclear ER and impaired ER 331 redistribution associated with PERK RNAi alone (fig. 6C and D). In accordance with 332 increased peripheral extension of sheet-like structures, CAMSAP2-depleted cells showed 333 higher signal density for RRBP1 immunostaining in the cell periphery (fig. 6E). It must be 334 noted that neither of these interventions affected significantly overall translation activity (fig. $335 \mathrm{~S} 5 \mathrm{C}$ ), suggesting that they do not provoke unspecific reduction of ER polysome density.

336 The most parsimonious explanation for these observations is that PERK deficiency leads to 337 a collapsed ER phenotype by stabilizing translation-dependent interactions between ER 338 shapers and non-centrosomal pools of MTs. 
339 We wondered whether ER stress and PERK activity might also affect specifically MTs 340 organization directly We appreciated in previous experiments that acute ER stress 341 induction was associated with the presence of prominent discrete MT nucleation centers 342 in wild-type cells, whereas PERK-depleted cells did not exhibit such features (see fig. $3436 \mathrm{~A}$, white arrowheads). To study this phenomenon in higher detail, we obtained MT 344 image sets using STED microscopy from cells exposed to acute ER stress, either 345 transfected with scrambled siRNA, or depleted for PERK, CAMSAP2, or both proteins 346 simultaneously (fig. 6F; see also fig. 6B). As expected, depletion of CAMSAP2 alone led 347 to a relative decrease in non-radial MT structures in the presence of vehicle indicating a 348 loss of non-centrosomal MTs(DMSO). Wild-type cells exhibited also decrease in non349 radial MT structures when exposed to acute ER stress, suggesting ER stress suppress 350 non-centrosomal MT polymerization. Of note, this phenotype correlated with a significant 351 decrease in CAMSAP2 total protein of c. $50 \%$, which was not observed in PERK352 depleted cells (see fig. 6B, lanes 2 vs 4). However, such decrease in non-centrosomal 353 MTs was not observed cells depleted for PERK (fig. 6F). These observations suggest 354 that PERK suppresses non-centrosomal MT polymerization. Finally, cells depleted of 355 both PERK and CAMSAP2 had very few non-centrosomal MTs suggesting PERK ability 356 to suppress non-centrosomal MT polymerization is upstream of CAMSAP2. Of note, 357 CAMSAP2 depletion attenuated tunicamycin-induced cell viability reduction in PERK358 deficient cells, albeit viability in DMSO-treated double-knockdown cells was also slightly 359 affected (fig. 6G). These observations support that non-centrosomal microtubules are 360 suppressed in a PERK-dependent fashion during ER stress to promote ER expansion. 361 Taken together with previous observations, this suggests that active polysomes allow for 362 the anchoring of ER shapers to non-centrosomal MTs in PERK-deficient cells, leading to 363 a collapsed ER structure upon ER stress induction.

\section{Disruption of PERK kinase activity impacts cell protrusiveness and motility}

365 Non-centrosomal MT arrays can regulate cell protrusiveness and migration ${ }^{41,43}$. Of note, 366 and as suggested by our initial screening image datasets, depletion of PERK kinase in 367 cells led to increased protrusion size but reduced protrusion number, and increased 368 polarity, as compared to wild-type cells (Fig. 7A-C). These observations are in agreement 369 with an interpretation that PERK activity could regulate cell polarity by suppressing the 370 polymerization of non-centrosomal MTs, which contribute to cell protrusiveness ${ }^{43}$. In 371 support of this idea, increased protrusiveness in PERK deficient cells was not observed in 372 PERK deficient cells also depleted for CAMSAP2 (Fig. 7A-C). 
374 To further assess the apparent directional protrusiveness phenotype of PERK-depleted 375 cells, we performed Transwell ${ }^{\mathrm{TM}}$ migration assays. We observed a higher degree of 376 protrusions passing through membrane pores when either depleting cells for the PERK

377 kinase using siRNA, as well as upon exposure to the PERK allosteric kinase inhibitor (fig.

378 7D, see also fig. S6A). Importantly, this increase in protrusiveness was abrogated by 379 simultaneous depletion of the non-centrosomal minus-end stabilizer CAMSAP2 (fig. 7D 380 and E). Increased polarized protrusiveness associated with PERK depletion was also 381 observed during neurite formation in neuroblasts (fig. 7): PERK-deficient cells exhibited 382 very long unique protrusions, as compared to wild type cells. Again, simultaneous 383 depletion of CAMSAP2 reverted these phenotypes.

384 We sought to study the correlation of these phenotypes with cell motility. We assessed the 385 impact of PERK signaling disruption on 3D cell migration. PERK inhibition enhanced cell 386 migration through a 3D collagen matrix, and this phenotype was inhibited upon depletion 387 of the non-centrosomal MT stabilizer CAMSAP2 (fig 7F). In accordance, cells plated on 388 soft collagen matrices exhibited long protrusions (fig. S6B). Of note, depletion of the ER389 MT linker RRBP1/p180 also reduced the increase in elongated protrusions and abrogated 390 the increase in 3D cell migration (fig. S6B and C). Artificial PERK activation in the 391 synthetic Fv2E-PEK model by exposure to the homodimerizer AP20178 had an opposite 392 effect on 3D cell migration, further supporting a role for PERK-dependent signaling on the 393 regulation of cell motility (fig. S6D). We propose a model whereby ER-MT reciprocal 394 regulation through PERK-dependent translation control has a dual impact on both 395 regulated ER remodeling, and non-centrosomal MT-dependent cell polarity and migration. 


\section{DISCUSSION:}

Eukaryotes evolved complex functional programs that continuously monitor ER

398 physical integrity and function and coordinate different responses in the cell to primarily

399 adapt its function, collectively termed Unfolded Protein Response (UPR). Here, we

400 contribute evidence that PERK-dependent control of protein translation modulates the

401 reciprocal regulation of $\mathrm{ER}$ and non-centrosomal microtubules, to both drive ER

402 remodeling during acute ER stress, and regulate non-centrosomal MT-dependent cell

403 protrusiveness and polarity.

404 Both ribosome association and microtubule cytoskeleton integrity can affect ER 405 architecture, as well as the localization of ER shapers ${ }^{29}$. Here, we demonstrate that this 406 relationship is controlled by the PERK kinase through elF2a phosphorylation-mediated 407 translation shutdown. ER architecture is tightly regulated by different 'shaper' proteins, 408 capable of defining different ER membrane domains ${ }^{2}$. The targeting of ER shaping 409 proteins must be controlled to allow for the dynamic remodeling of these membrane 410 structures for cell adaptation to different functional states, and different mechanisms have 411 been suggested, including posttranslational modifications and discrimination of differential 412 membrane curvature ${ }^{28,44-46}$. While a detailed picture of the precise mechanisms at play 413 requires further investigation, our results support that the control of the dynamics of 414 ternary complexes involving polysomes, microtubules and specific ER-anchored proteins, 415 is pivotal. The fact that depletion of each of the ER-MT linkers we have found in our study 416 ( 180 , Climp63 and REEP4) has a significant impact on the 'collapsed' phenotype we 417 observe in PERK-deficient cells suggests that each of these components is necessary, 418 but not sufficient, for the coupling of ER and MTs in epithelial cells. Given the impact of 419 these ER-MT tethers on MT network architecture (and particularly, on the non-radial 420 bundling of MTs when dysregulated $)^{25,28}$, it will be interesting to also explore the specific 421 role of each of these proteins on the dynamic stability of non-centrosomal MTs. An 422 additional pending question is the potential interplay of these mechanisms with the 423 reported crosstalk between the PERK-eIF2a axis and the actin cytoskeleton ${ }^{47-49}$; because 424 both cytoskeletons engage in mutual regulation ${ }^{50}$, it is plausible to assume their crosstalk 425 is relevant in the control of ER-MT coordination we report here.

Physical ER expansion and shape remodeling is a relevant adaptive event in 427 cells with compromised ER function (i.e. ER stress) ${ }^{4}$, and closely correlates with other 428 adaptive responses such as upregulation of protein maturation machineries ${ }^{51}$. PERK429 dependent coupling of attenuation of client protein load and ER expansion and remodeling 430 would ensure matching of both adaptive ER volume increase and protective translation 431 attenuation. Moreover, appropriate expansion of ER membrane might benefit from this 
432 coupling, ensuring a limited density of ER membrane-inserted proteins. At present, 433 despite the links with UPR signaling documented, we do not know what the relationship of 434 our findings with mechanisms driving ER membrane biogenesis is ${ }^{4,52}$. We have observed 435 that depletion of non-centrosomal microtubules attenuates the relative sensitivity of 436 PERK-deficient cells to acute ER stress, although CAMSAP2 depletion per se seems to 437 have an impact on ER homeostasis (see fig. 6). While the expansion of peripheral ER 438 sheets is an integral aspect of cell adaptation to ER stress, future studies will be required 439 to characterize in detail how architectural changes in the ER impact different outputs of 440 ER function. Additional pending questions for future exploration pertain to the precise 441 molecular mechanisms by which non-centrosomal MTs are regulated during ER stress 442 (figure 6). CAMSAP2 levels are indeed sensitive to acute ER stress induction ${ }^{53}$. Apart 443 from tight elF2a-dependent regulation of translation, other mechanisms coordinated by 444 PERK activation, such as autophagy ${ }^{54}$, may be at play

Available literature describes a relationship between ER architecture and MT 446 dynamics, and previous studies already suggested a specialization of ER-associated MT 447 pools $^{23}$. What is the significance for non-centrosomal MTs to determine ER architecture? 448 Functional segregation between centrosomal and non-centrosomal MTs is not fully 449 understood, but an emerging major role of non-centrosomal microtubules, as suggested 450 by studies manipulating this MT subpool, is the onset and maintenance of cell polarity and 451 protrusiveness across cell types ${ }^{41,43}$. Importantly, cell polarity and protrusiveness are 452 linked to organelle dynamics and trafficking through mechanisms that rely on the control 453 of organelle architecture, and couple them to de novo membrane synthesis ${ }^{55,56}$. It is 454 intuitive that their coupling to ER stress surveillance is convenient to ensure cell 455 homeostasis across functional states.

456 What is the physiopathological relevance of this dynamic link between ER and 457 non-centrosomal microtubules? Neuron differentiation and the control of neurite outgrowth 458 and axon stabilization is largely determined by the fine regulation of ER architecture, MT 459 organization and their reciprocal crosstalk therein ${ }^{57-59}$, and challenging ER homeostasis 460 can compromise this specialized form of cell protrusion stabilization ${ }^{60}$. Moreover, non461 centrosomal MTs appear to be specifically involved in these mechanisms ${ }^{57,61,62}$. Because 462 elF2a phosphorylation and PERK activity modulate memory stability and learning ${ }^{63-65}$, it 463 will be interesting to study the relevance of our findings in these contexts. Future studies 464 focusing on the impact of these mechanisms on directional vesicle trafficking and de novo 465 membrane formation, which seem to be relevant for protrusion formation, may also shed 466 light on these questions ${ }^{56}$. We have studied invasiveness phenotypes derived from PERK 467 depletion in mammary epithelial cells, as related to increased polarity. PERK activity has 
468 been studied in the context of mammary acini development and tumorigenesis, and its 469 complex impact on tumor cell survival and tissue organization ${ }^{35,66-68}$. Interestingly, recent 470 studies support a role for CAMSAP2 in tumor cell invasiveness ${ }^{69}$. The emerging picture is 471 complex, because the impact of intervening PERK signaling on tumor cells is highly 472 contextual and simultaneously affects different aspects of tumor cell biology: cell survival 473 and adaptation to nutrient deprivation and adverse environment, adhesion signaling and 474 accommodation of altered secretory phenotypes. Our results contribute a novel additional 475 perspective to this complex picture and suggest novel opportunities for synergistic 476 intervention of tumor cell biology by shutting down PERK-dependent prosurvival signaling 477 (autophagy, ROS management) and cell invasiveness.

478 
MATERIALS AND METHODS:

\section{Cell culture, transfection and reagents}

All cell maintenance and experiments were performed in a standard 483 humidified incubator at $37^{\circ} \mathrm{C}$ and $5 \% \mathrm{CO}_{2}$, unless otherwise stated. Low-passage 484 MCF10A cells, RasV12-transformed MCF10A ATI cells, and MCF10A-derived stable cell lines were cultured in DMEM-F12 Glutamax ${ }^{\circledR}$ medium (Gibco) supplemented, unless otherwise stated, with $5 \%$ heat-inactivated horse serum (Sigma), $1 \mu \mathrm{g} / \mathrm{ml}$ bovine insulin, $1 \mu \mathrm{M}$ hydrocortisone, $50 \mathrm{U}$ cholera toxin, and $100 \mathrm{ng} / \mathrm{ml}$ epidermal growth factor (EGF)- selection (puromycin, $5 \mathrm{ng} / \mathrm{ml}$ ) was applied in stable cell lines. HeLa, Sh-Sy5y and MDA-MB231 cells were grown in high glucose DMEM supplemented with $10 \%$ heat inactivated fetal bovine serum (Gibco).

491 MCF10A and MCF10A-ATI cells were a kind gift from Claire Isacke (ICR, UK); 492 MCF10A/Fv2E-PERK and MCF10A/PERKAC stable cell lines were generously provided 493 by Julio Aguirre-Ghiso (Mount Sinai Hospital, USA). ER bulk content was analyzed by flow 494 cytometry after brief pulse-labeling with BODIPY FL-ER tracker (Molecular Probes, 495 ThermoScientific) freshly resuspended cells after indicated treatments and analyzed on a 496 LSR Fortessa station. For siRNA reverse transfection, Lipofectamine RNAiMAX 497 (LifeSciences) reagent was used following supplier's recommendations. Plasmid 498 transfections were performed using Lipofectamine 3000 (LifeSciences) for 24h. 499 Tunicamycin, puromycin, sodium arsenite, cicloheximide, nocodazole, guanabenz and 500 Hoescht 33258 were obtained from Sigma. Thapsigargin was obtained from LifeSciences-

501 Invitrogen. The GSK202646 PERK inhibitor and harringtonin were purchased from Tocris.

502 AP20187 homodimerizer was purchased from Selleckchem, centrinone was purchased 503 from R\&D systems. Methanol-free, 16\% paraformaldehyde-PBS was purchased from

504 ThermoScientific. Secondary antibodies and fluorescent conjugates were purchased from 505 Molecular Probes. siRNAs were obtained from Dharmacon, esiRNAs were purchased 506 from Sigma. A table listing primary antibodies and siRNA/esiRNAs used is available as 507 supplementary table 1.

cDNA constructs, establishment of stable cell lines, and transient transfections MCF10A-EGFP-Sec61 $\beta$ cell line was obtained by lentiviral transduction (MOI:

511 5; Viral Vector unit, CNIC, Spain; lentiviral vector cloned on pRRL-IRES-mCherry 512 from Ndel/Mlul digested parent AcGFP-Sec61B vector Addgene \#15108) and two 513 subsequent rounds of stringent sorting (FACS Aria, BD Biosciences) of EGFP 514 positive cells with a fluorescence range within one order. Reverse transfection 
515 proceedings for transient silencing have been detailed elsewhere. For neurite 516 extension assays, SH-Sy5y cells lentivirally transduced with a pRRL-IRES-EGFP 517 vector (MOI: 1; Viral Vector unit, CNIC, Spain) were reverse transfected with 518 indicated esiRNAs and incubated for $48 \mathrm{~h}$, then switched to differentiation medium $519(1.5 \% \mathrm{FBS}, 1 \mu \mathrm{g} / \mathrm{ml}$ bovine insulin, $1 \mu \mathrm{M}$ hydrocortisone, $10 \mu \mathrm{M}$ trans-retinoic acid 520 (Sigma $\AA)$, and $5 \mathrm{U}$ brain-derived neurotropic factor (BDNF, Sigma®)) and incubated 521 for a further $48 \mathrm{~h}$ before being processed for immunofluorescence. elF2a WT and 522 S51D constructs were cloned on pcDNA3.1-HA from Addgene constructs \#21807 523 and \#21809. In experiments detailed in figure 4A, where simultaneous transfection of 524 cDNA constructs was required, cells were reverse transfected as detailed above, and $52524 \mathrm{~h}$ after directly transfected using the Lipofectamine 3000 reagent.

\section{High-throughput assays, immunostaining and acquisition}

528 For high content imaging, cells were reverse transfected using Lipofectamine RNAiMAX 529 reagent, and 40ng of siRNA, on optical CellCarrier 384-well plates, on a final volume of $53040 \mu \mathrm{l}$. Liquid handling, fixation and immunostaining was performed using a robotic station

531 (HCS Explorer, PerkinElmer) according to previously detailed protocols ${ }^{70}$. Acquisition and 532 automated image analysis was performed with an Opera HCS II spinning disk confocal 533 microscope.

\section{Superesolution confocal microscopy and transmission electron microscopy (TEM)}

536 A Leica SP8 3X STED spectral confocal microscope, equipped with hybrid 537 photomultipliers, a CCD digital camera and a STED station with two separate depletion 538 lasers (592 and 660nm) was used, with a 100X/1.4NA immersion objective. Sequential 539 (between stacks) acquisition used 100\% power for STED depletion and $70 \%$ power for 540 illumination. Samples were prepared according to the manufacturer's recommendations.

541 For TEM, cells grown on $100-\mathrm{mm}$ dishes treated as indicated were fixed with $4 \%$ 542 paraformaldehyde and $2 \%$ glutaraldehyde for $120 \mathrm{~min}$ at room temperature. Upon gentle 543 scrapping, postfixation was carried out with $1 \% \mathrm{OsO} 4$ and $1.0 \% \mathrm{~K} 3 \mathrm{Fe}(\mathrm{CN}) 6$ in $\mathrm{H} 2 \mathrm{O}$ at $5444^{\circ} \mathrm{C}$ for $60 \mathrm{~min}$. Samples were dehydrated with ethanol and embedded in Epoxy, TAAB 545812 Resin (TAAB Laboratories) according to standard procedures. Ultrathin $(80 \mathrm{~nm})$ 546 sections were stained with saturated uranyl acetate and lead citrate and visualized with a 547 JEOL JEM 1010 (Tokyo, Japan) electron microscope at $80 \mathrm{kV}$. 16-bit images were 
548 recorded with a 4 k $\times 4$ k CMOS F416 camera from TVIPS (Gauting, Germany), typically

549 at $12000 \times$ magnification.

550

\section{Image analysis}

552 Image analysis proceedings are detailed together with full script file as supplementary

553 data, and comprehended the following steps: (1) nuclei and cell segmentation; (2) filtering

554 of artifacts and out-of-focus objects; (3) definition of subcytoplasmic regions, and (4)

555 gathering of ER-related features. ImageJ analysis pipelines used in experiments shown in

556 figures 6 and 7 have been previously reported ${ }^{43}$.

\section{Protein analysis}

559 Cell lysates were harvested in colorless, non-reducing sample buffer ( $2 \%$ SDS, $150 \mathrm{KCl}$,

$56020 \mathrm{mM}$ Tris pH6.8, 10\% glycerol, protease and phosphatase inhibitors), quantitated

561 through Bradford assay and normalized, and supplemented with DTT and bromophenol

562 blue to a final concentration of $0.2 \mathrm{mM}$ and $0.012 \%$, respectively. $\sim 10 \mu \mathrm{g} / \mathrm{sample}$ were

563 loaded on 10-12.5\% SDS-polyacrylamide gels. After electrophoretic separation and

564 blotting in conventional non-SDS, $20 \%$ methanol conditions to PVDF membranes,

565 samples were probed with the indicated antibodies according to standard protocols ${ }^{31}$.

566 Signal was developed with the ECL Plus system (PerkinElmer).

\section{Puromycilation assay}

569 Our protocol was a slight modification of procedures published previously ${ }^{37}$. Briefly, cells 570 to be labeled were exposed in a $\sim 45$ " pulse to a low concentration of puromycin $571(500 \mathrm{ng} / \mathrm{ml})$ and immediately washed twice in complete medium and fixed with $4 \%$ PFA. 572 Samples were permeabilized $15 \mathrm{~min}$ in $0.2 \%$ triton-X100 PBS, washed twice in $0.05 \%$ TX-

573100 PBS, and blocked for $2 \mathrm{~h}$ with $2 \%$ BSA in PBS. Standard immunofluorescence 574 procedures were thereon applied to label with the 2B4 anti-puromycin monoclonal 575 antibody, developed by $A$. David and coworkers and publicly available through the 576 Developmental Studies Hybridoma Bank repository (entry \#PMY-24B)

\section{Cell viability assays}

579 Cell viability was inferred by the MTT colorimetric assay. Briefly, cells were treated with 580 either vehicle (DMSO) or tunicamycin $(5 \mu \mathrm{g} / \mathrm{ml}, 36 \mathrm{~h}$ ), and then exposed to $50 \mu \mathrm{g} / \mathrm{ml}$ MTT 
581 (3-(4,5-dimethylthiazol-2-yl)-2,5-diphenyltetrazolium bromide at $37{ }^{\circ} \mathrm{C}$ for 3 hours in the

582 dark. Precipitated formazan was solubilized in DMSO, and absorption was measured at

$583542 \mathrm{~nm}$ in a spectrophotometer. Absorption values were referred to control condition as

$584100 \%$.

585

586 Transwell migration assays

$5878.0 \mu \mathrm{m} \varnothing$ - pore PET hanging inserts were pre-coated with collagen as recommended by

588 the supplier (Millipore). Cells were seeded in a $500 \mu$ l volume of reverse transfection 589 mixture and allowed to migrate for $\sim 4 \mathrm{~h}$. Inserts were then placed on pre-warmed $4 \%$ PFA-

590 PBS, and processed for immunostaining following standard protocols. Images were

591 acquired on a Zeis Axiovert confocal microscope. Basal (trans) fluorescence was related

592 to top (cis) fluorescence from the actin channel as segmented and quantitated by ImageJ

593 standard tools from raw .lif images.

594

\section{Culture on collagen matrices}

$596 \sim 100 \mu \mathrm{l}$ Fibrillar dermal bovine collagen I layers $(1.7 \mathrm{mg} / \mathrm{ml})$ were casted on 96-well

597 optical ViewPlate plates (PerkinElmer), following previously detailed protocols. $\sim 4000$

598 (MCF10A, MCF10-ATI) or 6500 (MDA-MB231) cells were reverse transfected and plated

599 on top of the pre-casted collagen plugs, as described, on a total volume of $50 \mu \mathrm{l}$ of

600 complete medium. After 48h, cells were pulse-labelled with CellTracker Orange 561

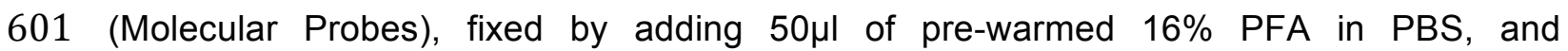

602 counterstained by adding 10ng of Hoeschtt 33342. Acquisition of focal stacks was

603 performed on an Opera HCS II spinning disk microscope, and in-focus images were 604 manually selected for further analysis.

605

606 3D Collagen invasion assays

607 96-well optical glass plates (PerkinElmer) were coated overnight at 37oC with an sterile $6081 \%$ solution of BSA to minimize cell attachment. Cells reverse transfected for $24 \mathrm{~h}$ were 609 detached and embedded in a $1.7 \mathrm{mg} / \mathrm{ml}$ fibrillar dermal bovine collagen I matrix at a 610 density of $50000 \mathrm{cells} / \mathrm{ml}$, and $150 \mu \mathrm{l}$ were plated per well. Plates were immediately spun at $611300 \mathrm{~g}$ for $5 \mathrm{~min}$ and incubated for $\sim 4 \mathrm{~h}$ at $37^{\circ} \mathrm{C}$ to allow for collagen polymerization. $50 \mu \mathrm{l}$ 612 completion media ( $4 \mathrm{X}$ growth medium) containing the indicated treatments was added, 613 and cells were allowed to invade the matrix overnight. Subsequently, samples were fixed 614 and counterstained adding $50 \mu$ l of $16 \%$ PFA in PBS containing $500 \mathrm{ng} / \mathrm{ml}$ of Hoeschtt 
615 33342. Plates were then imaged using an Operetta HCS system (PerkinElmer; ICR, 616 London, UK), acquiring three optical planes with a spacing of $\sim 50 \mu \mathrm{m}$. Nuclei segmentation 617 and counting per plane was performed using the Columbus automated system 618 (PerkinElmer).

619

620

621

622

623

624

625

626

627

628

629

630

631

632

633 
634 ACKOWLEDGEMENTS: For sharing with us valuable reagents and/or suggestions, 635 we are grateful to professors Rachael Natrajan, Francisco Sánchez-Madrid, Julio 636 Aguirre-Ghisso, Hesso Farhan, Françoise van der Goot and Jonathan Yewdell. We 637 acknowledge the efficient and friendly assistance of the following core services: Flow 638 Cytometry core facility at the ICR (London, UK), Advanced Light Microscopy at CNIC 639 (Madrid, Spain), and Electron Microscopy at CBM-CSIC (Madrid, Spain). We are 640 deeply grateful to the Cellomics Unit at CNIC for assistance and infrastructure, and to 641 other members of the Bakal and the del Pozo labs for their input and advice. Amine 642 Sadok and Faraz Mardakheh (former researchers at ICR, London, UK) provided 643 expert advice and assistance on collagen migration and Transwell ${ }^{\mathrm{TM}}$ experiments. 644 Where indicated, plasmid and antibody resources were obtained from the Addgene 645 and DSHB public repositories and we are grateful for their excellent non-profit 646 service. $\mathrm{CB}$ and HS have been beneficiaries of the Wellcome Trust Career 647 Development Fellowship program. MS-A was a fellow of the COFUND-IPP 648 programme (2014). Funding support was received from the Cancer Research UK 649 (CRUK) Programme Foundation Award (C37275/A20146) and the Stand Up to 650 Cancer campaign for Cancer Research UK to CB; and from the spanish Ministerio de 651 Ciencia e Innovación (MCNU; SAF2017-83130-R and BFU2016-81912-REDC), the 652 Comunidad Autónoma de Madrid/FEDER, Spain (ref. S2018/NMT4443; Actividades 653 de I+D entre Grupos de Investigación en Tecnologías) and the Fundació La Marató 654 de TV3 $(385 / \mathrm{C} / 2019)$ to MAdP. The CNIC is supported by the Instituto de Salud 655 Carlos III (ISCIII), the Ministerio de Ciencia, Innovación y Universidades (MCNU) and 656 the Pro CNIC Foundation, and is a Severo Ochoa Center of Excellence (SEV-2015657 0505).

658

659 AUTHOR CONTRIBUTIONS: MS-A and CB conceived and designed the study. HS 660 provided image analysis tools for initial screen. MS-A performed experimental work and 661 analyzed data, with assistance from FNL and GF. PP-V and MA-G acquired relevant 662 preliminary results. MAdP provided key resources and advice. MS-A and CB wrote the 663 paper. 
bioRxiv preprint doi: https://doi.org/10.1101/2021.01.19.426991; this version posted January 19, 2021. The copyright holder for this preprint (which was not certified by peer review) is the author/funder. All rights reserved. No reuse allowed without permission.

668 
669

670

671

672

673

674

675

676

677

678

679

680

681

682

683

684

685

686

687

688

689

690

691

692

693

694

695

696

697

698

699

\section{REFERENCES:}

1. Braakman, I. \& Bulleid, N. J. Protein Folding and Modification in the Mammalian Endoplasmic Reticulum. Annu. Rev. Biochem. (2011). doi:10.1146/annurev-biochem-062209-093836

2. Shibata, Y., Hu, J., Kozlov, M. M. \& Rapoport, T. A. Mechanisms Shaping the Membranes of Cellular Organelles. Annu. Rev. Cell Dev. Biol. (2009). doi:10.1146/annurev.cellbio.042308.113324

3. Westrate, L. M., Lee, J. E., Prinz, W. A. \& Voeltz, G. K. Form Follows Function: The Importance of Endoplasmic Reticulum Shape. Annu. Rev. Biochem. 84, 791-811 (2015).

4. Schuck, S., Prinz, W. A., Thorn, K. S., Voss, C. \& Walter, P. Membrane expansion alleviates endoplasmic reticulum stress independently of the unfolded protein response. J. Cell Biol. (2009). doi:10.1083/jcb.200907074

5. Karagöz, G. E., Acosta-Alvear, D. \& Walter, P. The unfolded protein response: Detecting and responding to fluctuations in the protein-folding capacity of the endoplasmic reticulum. Cold Spring Harb. Perspect. Biol. (2019). doi:10.1101/cshperspect.a033886

6. Volmer, R., Van Der Ploeg, K. \& Ron, D. Membrane lipid saturation activates endoplasmic reticulum unfolded protein response transducers through their transmembrane domains. Proc. Natl. Acad. Sci. U. S. A. (2013). doi:10.1073/pnas.1217611110

7. Walter, P. \& Ron, D. The unfolded protein response: From stress pathway to homeostatic regulation. Science (2011). doi:10.1126/science.1209038

8. Korennykh, A. \& Walter, P. Structural Basis of the Unfolded Protein Response. Annu. Rev. Cell Dev. Biol. (2012). doi:10.1146/annurevcellbio-101011-155826

9. Tam, A. B. et al. The UPR Activator ATF6 Responds to Proteotoxic and Lipotoxic Stress by Distinct Mechanisms. Dev. Cell (2018). doi:10.1016/j.devcel.2018.04.023 
700

701

702

703

704

705

706

707

708

709

710

711

712

713

714

715

716

717

718

719

720

721

722

723

724

725

726

727

728

729

730

10. Bommiasamy, $\mathrm{H}$. et al. ATF6a induces XBP1-independent expansion of the endoplasmic reticulum. J. Cell Sci. (2009). doi:10.1242/jcs.045625

11. Sriburi, R., Jackowski, S., Mori, K. \& Brewer, J. W. XBP1: A link between the unfolded protein response, lipid biosynthesis, and biogenesis of the endoplasmic reticulum. J. Cell Biol. (2004). doi:10.1083/jcb.200406136

12. Sriburi, R. et al. Coordinate regulation of phospholipid biosynthesis and secretory pathway gene expression in XBP-1(S)-induced endoplasmic reticulum biogenesis. J. Biol. Chem. (2007). doi:10.1074/jbc.M609490200

13. Harding, H. P., Zhang, Y., Bertolotti, A., Zeng, H. \& Ron, D. Perk is essential for translational regulation and cell survival during the unfolded protein response. Mol. Cell (2000). doi:10.1016/S10972765(00)80330-5

14. Harding, H. P., Calfon, M., Urano, F., Novoa, I. \& Ron, D. Transcriptional and Translational Control in the Mammalian Unfolded Protein Response. Annu. Rev. Cell Dev. Biol. (2002). doi:10.1146/annurev.cellbio.18.011402.160624

15. Harding, H. P. et al. Regulated translation initiation controls stressinduced gene expression in mammalian cells. Mol. Cell (2000). doi:10.1016/S1097-2765(00)00108-8

16. Verfaillie, T. et al. PERK is required at the ER-mitochondrial contact sites to convey apoptosis after ROS-based ER stress. Cell Death Differ. (2012). doi:10.1038/cdd.2012.74

17. Gurel, P. S., Hatch, A. L. \& Higgs, H. N. Connecting the cytoskeleton to the endoplasmic reticulum and Golgi. Current Biology (2014). doi:10.1016/j.cub.2014.05.033

18. Griffing, L. R. Networking in the endoplasmic reticulum. in Biochemical Society Transactions (2010). doi:10.1042/BST0380747

19. Prinz, W. A. et al. Mutants affecting the structure of the cortical endoplasmic reticulum in Saccharomyces cerevisiae. J. Cell Biol. 
732

733

20. Puhka, M., Joensuu, M., Vihinen, H., Belevich, I. \& Jokitalo, E. Progressive sheet-to-tubule transformation is a general mechanism for endoplasmic reticulum partitioning in dividing mammalian cells. Mol. Biol. Cell (2012). doi:10.1091/mbc.E10-12-0950

21. Puhka, M., Vihinen, H., Joensuu, M. \& Jokitalo, E. Endoplasmic reticulum remains continuous and undergoes sheet-to-tubule transformation during cell division in mammalian cells. J. Cell Biol. (2007). doi:10.1083/jcb.200705112

22. Terasaki, M., Chen, L. B. \& Fujiwara, K. Microtubules and the endoplasmic reticulum are highly interdependent structures. J. Cell Biol. (1986). doi:10.1083/jcb.103.4.1557

23. Friedman, J. R., Webster, B. M., Mastronarde, D. N., Verhey, K. J. \& Voeltz, G. K. ER sliding dynamics and ER-mitochondrial contacts occur on acetylated microtubules. J. Cell Biol. (2010). doi:10.1083/jcb.200911024

24. Grigoriev, I. et al. STIM1 Is a MT-Plus-End-Tracking Protein Involved in Remodeling of the ER. Curr. Biol. (2008). doi:10.1016/j.cub.2007.12.050

25. Ogawa-Goto, K. et al. p180 is involved in the interaction between the endoplasmic reticulum and microtubules through a novel microtubulebinding and bundling domain. Mol. Biol. Cell (2007). doi:10.1091/mbc.E06-12-1125

26. Park, S. H., Zhu, P. P., Parker, R. L. \& Blackstone, C. Hereditary spastic paraplegia proteins REEP1, spastin, and atlastin-1 coordinate microtubule interactions with the tubular ER network. J. Clin. Invest. (2010). doi:10.1172/JCl40979

27. Rodríguez-Feo, J. A., Gallego-Delgado, J., Puerto, M., Wandosell, F. \& Osende, J. Reticulon-4B/Nogo-B acts as a molecular linker between microtubules and actin cytoskeleton in vascular smooth muscle cells. Biochim. Biophys. Acta - Mol. Cell Res. (2016). 
763

764

765

766

767

768

28. Vedrenne, C., Klopfenstein, D. R. \& Hauri, H. P. Phosphorylation controls CLIMP-63-mediated anchoring of the endoplasmic reticulum to microtubules. Mol. Biol. Cell (2005). doi:10.1091/mbc.E04-07-0554

29. Shibata, Y. et al. Mechanisms determining the morphology of the peripheral ER. Cell (2010). doi:10.1016/j.cell.2010.11.007

30. Hu, J., Prinz, W. A. \& Rapoport, T. A. Weaving the web of ER tubules. Cell (2011). doi:10.1016/j.cell.2011.11.022

31. Sanchez-Alvarez, M., Del Pozo, M. A. \& Bakal, C. AKT-mTOR signaling modulates the dynamics of IRE1 RNAse activity by regulating ERmitochondria contacts. Sci. Rep. (2017). doi:10.1038/s41598-01716662-1

32. Sanchez-Alvarez, M., Zhang, Q., Finger, F., Wakelam, M. J. O. \& Bakal, C. Cell cycle progression is an essential regulatory component of phospholipid metabolism and membrane homeostasis. Open Biol. (2015). doi:10.1098/rsob.150093

33. Shaffer, A. L. et al. XBP1, downstream of Blimp-1, expands the secretory apparatus and other organelles, and increases protein synthesis in plasma cell differentiation. Immunity (2004). doi:10.1016/j.immuni.2004.06.010

34. Avivar-Valderas, A. et al. PERK Integrates Autophagy and Oxidative Stress Responses To Promote Survival during Extracellular Matrix Detachment. Mol. Cell. Biol. (2011). doi:10.1128/mcb.05164-11

35. Sequeira, S. J. et al. Inhibition of proliferation by PERK regulates mammary acinar morphogenesis and tumor formation. PLoS One (2007). doi:10.1371/journal.pone.0000615

36. Bobrovnikova-Marjon, E. et al. PERK-dependent regulation of lipogenesis during mouse mammary gland development and adipocyte differentiation. Proc. Natl. Acad. Sci. U. S. A. (2008). doi:10.1073/pnas.0808517105

37. David, A. et al. Nuclear translation visualized by ribosome-bound 
nascent chain puromycylation. J. Cell Biol. (2012).

38. Tsaytler, P., Harding, H. P., Ron, D. \& Bertolotti, A. Selective inhibition of a regulatory subunit of protein phosphatase 1 restores proteostasis. Science (80-. ). (2011). doi:10.1126/science.1201396

39. Hollien, J. Evolution of the unfolded protein response. Biochim. Biophys. Acta - Mol. Cell Res. 1833, 2458-2463 (2013).

40. Kumar, D., Golchoubian, B., Belevich, I., Jokitalo, E. \& Schlaitz, A. L. REEP3 and REEP4 determine the tubular morphology of the endoplasmic reticulum during mitosis. Molecular Biology of the Cell (2019). doi:10.1091/mbc.E18-11-0698

41. Martin, M. \& Akhmanova, A. Coming into Focus: Mechanisms of Microtubule Minus-End Organization. Trends in Cell Biology (2018). doi:10.1016/j.tcb.2018.02.011

42. Wong, Y. L. et al. Reversible centriole depletion with an inhibitor of Polo-like kinase 4. Science (80-. ). (2015).

doi:10.1126/science.aaa5111

43. Martin, M., Veloso, A., Wu, J., Katrukha, E. A. \& Akhmanova, A. Control of endothelial cell polarity and sprouting angiogenesis by noncentrosomal microtubules. Elife (2018). doi:10.7554/eLife.33864

44. Zhao, Y., Zhang, T., Huo, H., Ye, Y. \& Liu, Y. Lunapark is a component of a ubiquitin ligase complex localized to the endoplasmic reticulum three-way junctions. J. Biol. Chem. (2016). doi:10.1074/jbc.M116.737783

45. Wang, X., Li, S., Wang, H., Shui, W. \& Hu, J. Quantitative proteomics reveal proteins enriched in tubular endoplasmic reticulum of Saccharomyces cerevisiae. Elife (2017). doi:10.7554/eLife.23816

46. Wang, N. \& Rapoport, T. A. Reconstituting the reticular ER network Mechanistic implications and open questions. Journal of Cell Science (2019). doi:10.1242/jcs.227611

47. Chen, R. et al. G-actin provides substrate-specificity to eukaryotic 
initiation factor 2alpha holophosphatases. Elife 4, (2015).

825

48. Chambers, J. E. et al. Actin dynamics tune the integrated stress response by regulating eukaryotic initiation factor 2alpha dephosphorylation. Elife 4, (2015).

49. van Vliet, A. R. et al. The ER Stress Sensor PERK Coordinates ERPlasma Membrane Contact Site Formation through Interaction with Filamin-A and F-Actin Remodeling. Mol. Cell 65, 885-899.e6 (2017).

50. Colin, A., Singaravelu, P., Thery, M., Blanchoin, L. \& Gueroui, Z. Actin-

51. Bakunts, A. et al. Ratiometric sensing of BiP-client versus BiP levels by the unfolded protein response determines its signaling amplitude. Elife 6, (2017).

52. Bircham, P. W. et al. Control of endoplasmic reticulum membrane biogenesis by regulators of lipid metabolism. bioRxiv 2020.02.23.961722 (2020). doi:10.1101/2020.02.23.961722

53. Itzhak, D. N. et al. SILAC-based quantitative proteomics using mass

54. Kim, K. W., Moretti, L., Mitchell, L. R., Jung, D. K. \& Lu, B. Endoplasmic spectrometry quantifies endoplasmic reticulum stress in whole HeLa cells. Dis. Model. Mech. 12, (2019).

55. Zhang, C., Lu, J., Su, H., Yang, J. \& Zhou, D. Fatty acid synthase reticulum stress mediates radiation-induced autophagy by perkelF2alpha in caspase-3/7-deficient cells. Oncogene 29, 3241-3251 (2010).

56. Raiborg, C. et al. Repeated ER-endosome contacts promote endosome translocation and neurite outgrowth. Nature (2015). doi:10.1038/nature14359

853 57. Farías, G. G. et al. Feedback-Driven Mechanisms between 854 Microtubules and the Endoplasmic Reticulum Instruct Neuronal Polarity. 
58. Kapitein, L. C. \& Hoogenraad, C. C. Building the Neuronal Microtubule

59. Cui-Wang, T. et al. Local zones of endoplasmic reticulum complexity confine cargo in neuronal dendrites. Cell (2012). doi:10.1016/j.cell.2011.11.056

60. Yavin, E., Richter-Landsberg, C., Duksin, D. \& Yavin, Z. Tunicamycin blocks neuritogenesis and glucosamine labeling of gangliosides in developing cerebral neuron cultures. Proc. Natl. Acad. Sci. U. S. A. 81, 5638-5642 (1984).

61. Pongrakhananon, V. et al. CAMSAP3 maintains neuronal polarity through regulation of microtubule stability. Proc. Natl. Acad. Sci. U. S.

62. Yau, K. W. et al. Microtubule minus-end binding protein CAMSAP2

63. Chou, A. et al. Inhibition of the integrated stress response reverses cognitive deficits after traumatic brain injury. Proc. Natl. Acad. Sci. U. S.

64. Sharma, V. et al. Local inhibition of PERK enhances memory and reverses age-related deterioration of cognitive and neuronal properties. J. Neurosci. (2018). doi:10.1523/JNEUROSCI.0628-17.2017

65. Sharma, V. et al. elF2 $\alpha$ controls memory consolidation via excitatory and somatostatin neurons. Nature 586, 412-416 (2020).

66. Feng, Y. X. et al. Cancer-specific PERK signaling drives invasion and metastasis through CREB3L1. Nat. Commun. (2017).

882 67. Del Vecchio, C. A. et al. De-Differentiation Confers Multidrug 
885 68. Feng, Y. X. et al. Epithelial-to-mesenchymal transition activates PERKelF2 $\alpha$ and sensitizes cells to endoplasmic reticulum stress. Cancer Discov. (2014). doi:10.1158/2159-8290.CD-13-0945

69. Li, D. et al. CAMSAP2-mediated noncentrosomal microtubule acetylation drives hepatocellular carcinoma metastasis. Theranostics 10, 3749-3766 (2020).

70. Sanchez-Alvarez, M., Del Pozo, M. A. \& Bakal, C. AKT-mTOR signaling modulates the dynamics of IRE1 RNAse activity by regulating ERmitochondria contacts. Sci. Rep. 7, (2017).

894 
bioRxiv preprint doi: https://doi.org/10.1101/2021.01.19.426991; this version posted January 19, 2021. The copyright holder for this preprint (which was not certified by peer review) is the author/funder. All rights reserved. No reuse allowed without permission.

A
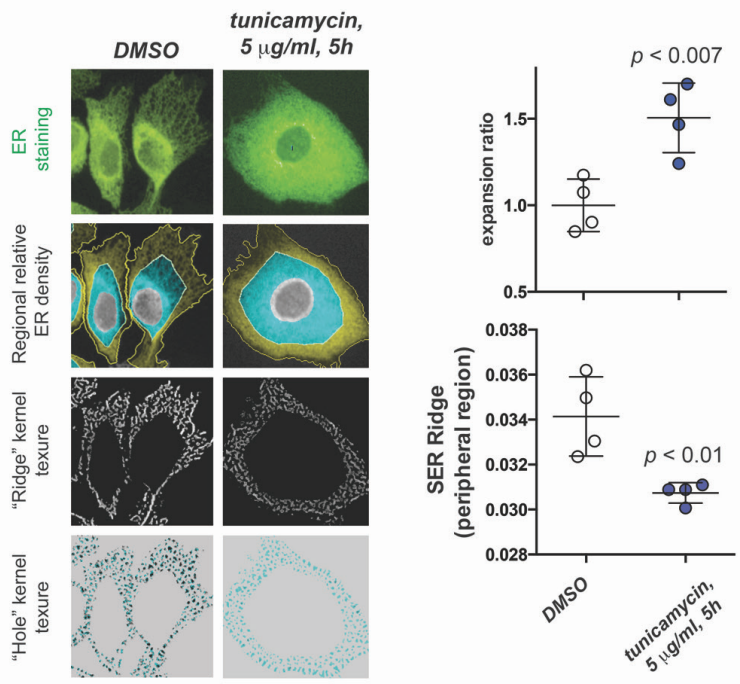

B

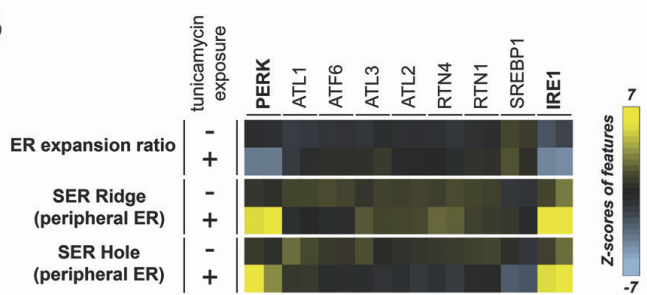

C

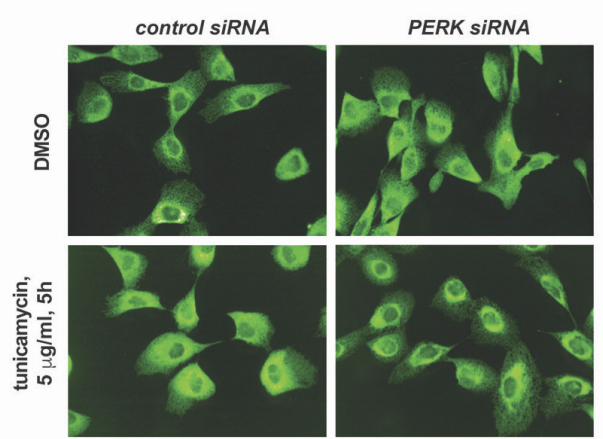

D

ODMSO

O tunicamycin, $5 \mu \mathrm{g} / \mathrm{ml}$, $5 \mathrm{~h}$
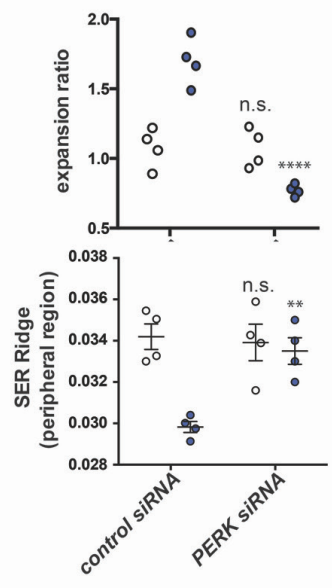

E
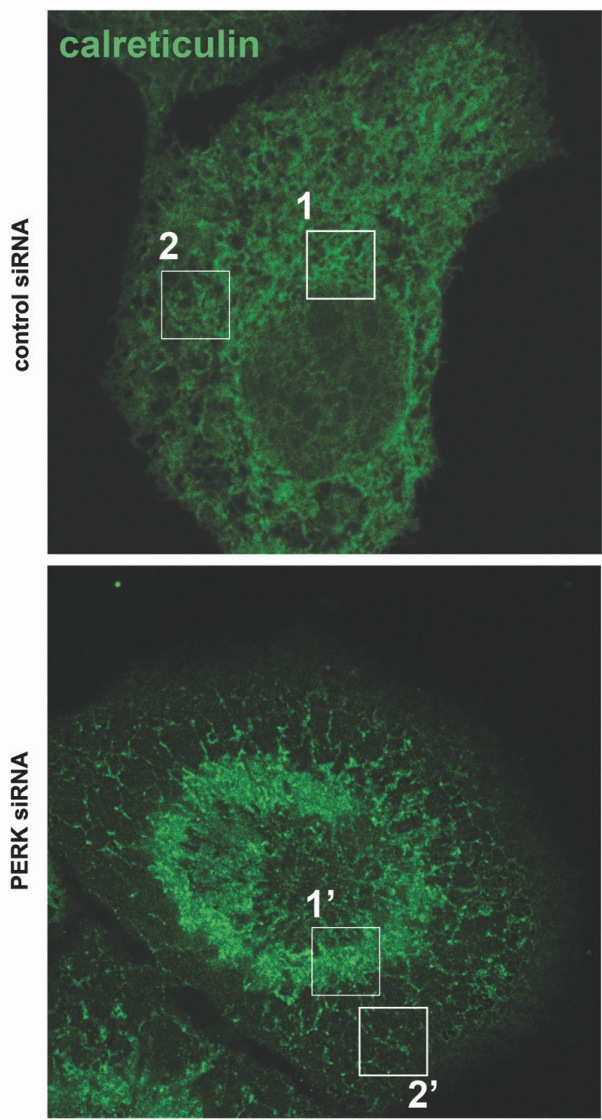

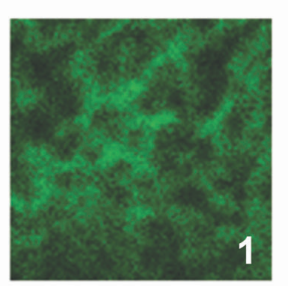

1
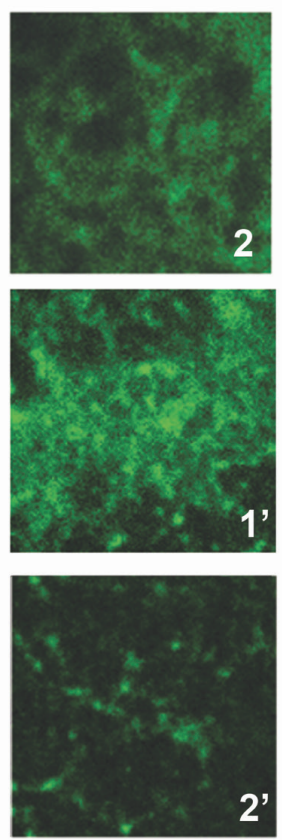

$\mathbf{F}$
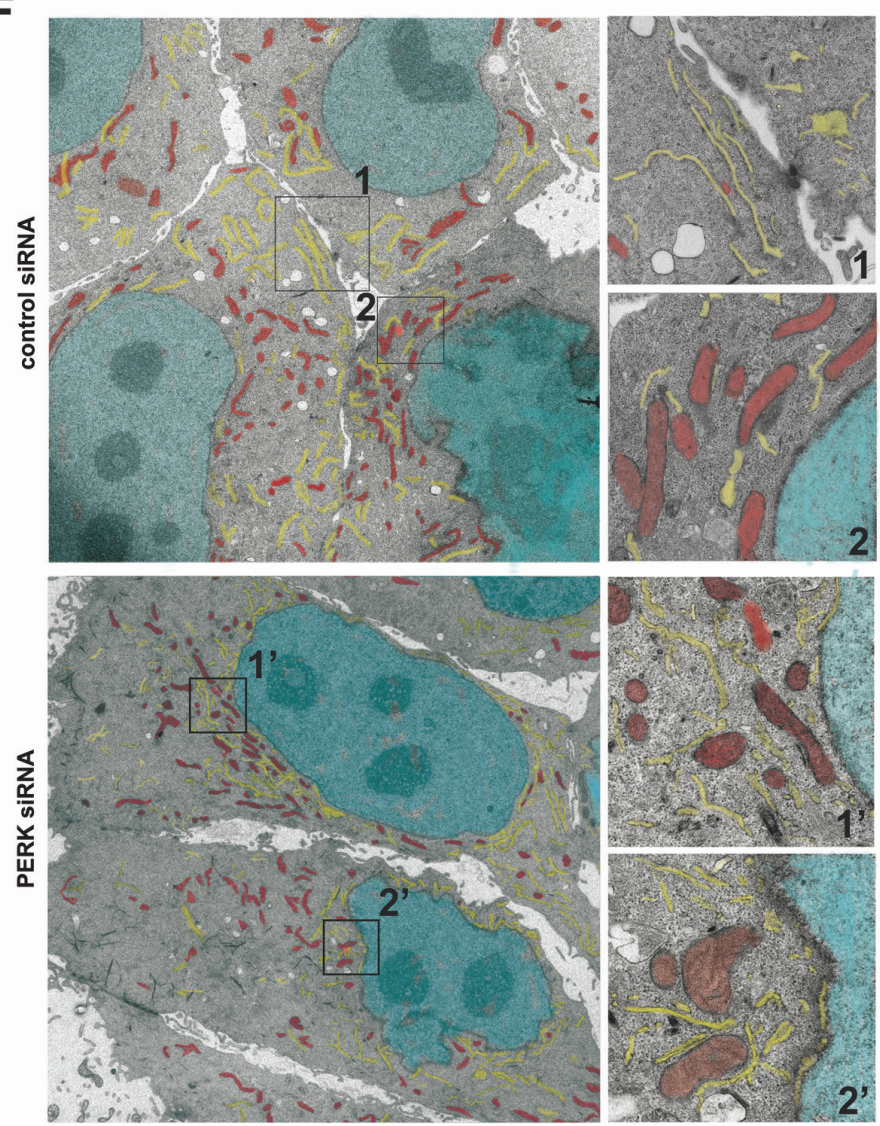

896

897 Figure 1.- An automated image-based assay identifies EIF2AK3/PERK as

898 required for ER remodeling during acute ER stress. (A) Examples of

899 automated image analysis features informative of ER architecture remodeling

900 in MCF10A cells treated as indicated, from unprocessed immunofluorescence

901 images of total ER (anti-calreticulin immunostaining). Graphs (rightmost

902 panels) are derived from four independent replicates of indicated conditions.

903 (B-D) Results from automated image-based exploration of genetic regulators 
904 of ER stress-driven ER remodeling, using pools of 4 siRNA duplexes to 905 interrogate each chosen gene. [B] Heatmaps (Z-scores) of selected features, 906 informative for ER remodeling, across conditions, as compared to control 907 cells. [C] Representative images for indicated conditions. [D] Graphs derived 908 from well-normalized values for indicated features across conditions (four 909 independent replicates. (E, F) STED microscopy (calreticulin immunostaining) $910[\mathrm{E}]$ and electron microscopy [F] of MCF10A cells exposed for $6 \mathrm{~h}$ to $5 \mu \mathrm{g} / \mathrm{ml}$ of 911 tunicamycin. Magnified cropped images show details of indicated ROls at 912 perinuclear or peripheral cell regions. Pseudocolouring in [F]: cyan- nuclei; 913 red-mitochondria; yellow-ER. $p$ values are indicated across experiments. 914 Statistical significance across assays was assessed by paired t-Student test; 915 *: $p<0.05 ;{ }^{* *}: p<0.01$; ${ }^{* * *}: p<0.005$. n.s.: $p>0.05$ 
bioRxiv preprint doi: https://doi.org/10.1101/2021.01.19.426991; this version posted January 19, 2021. The copyright holder for this

A

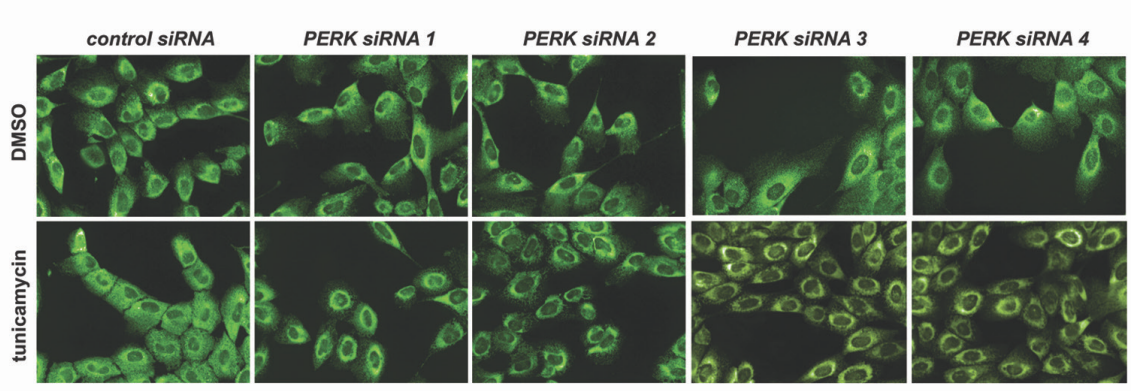

C
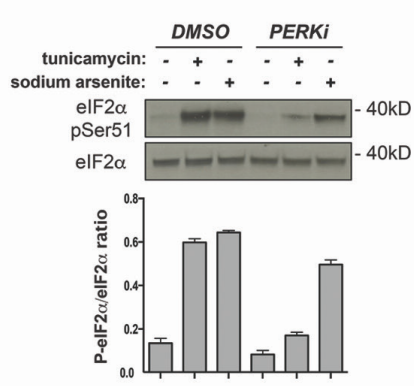

$\mathbf{F}$
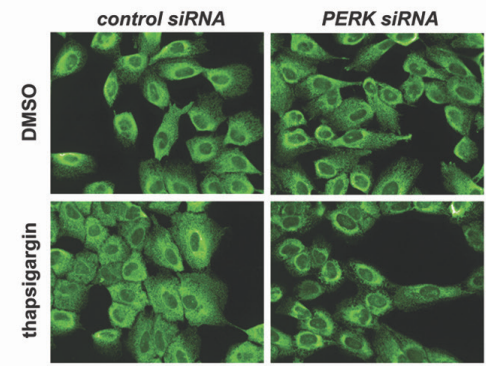

H

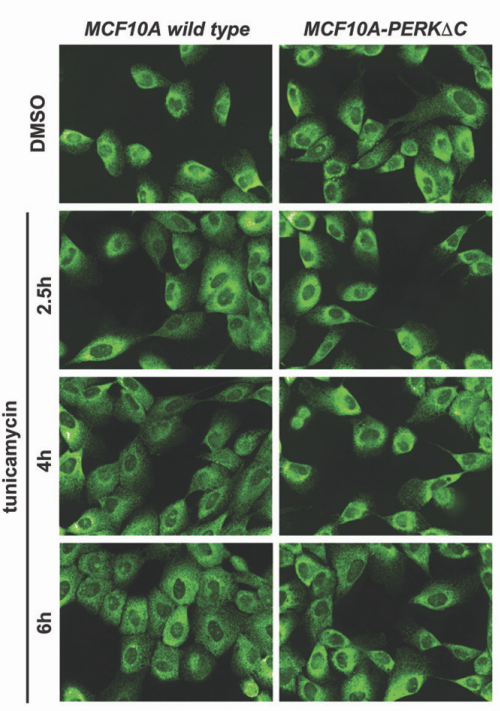

D

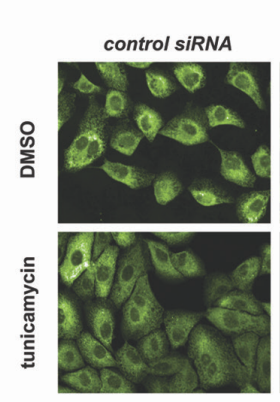

G

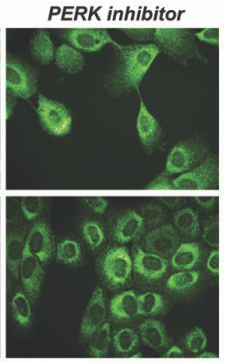

I

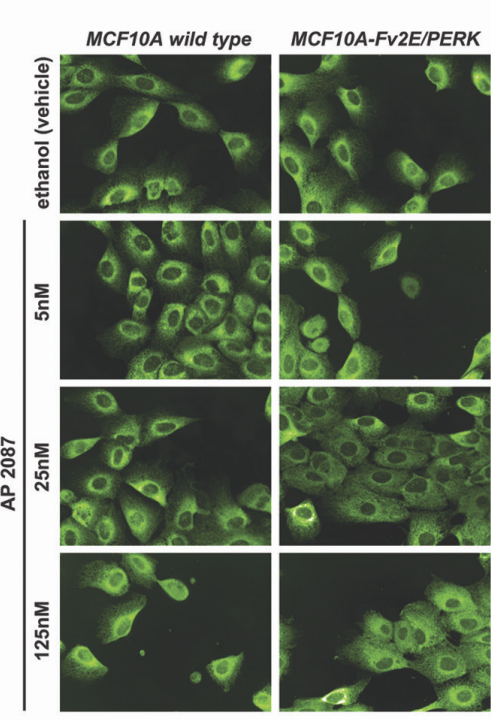

$\mathrm{B}$

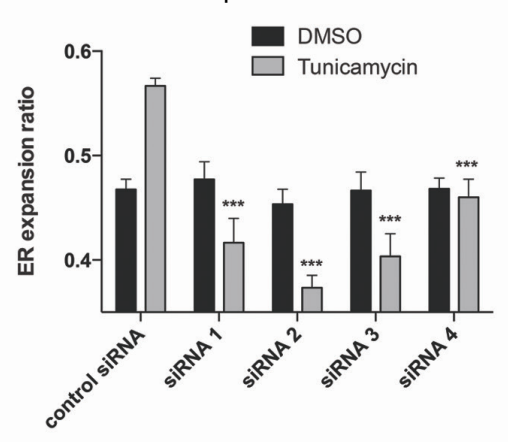

E

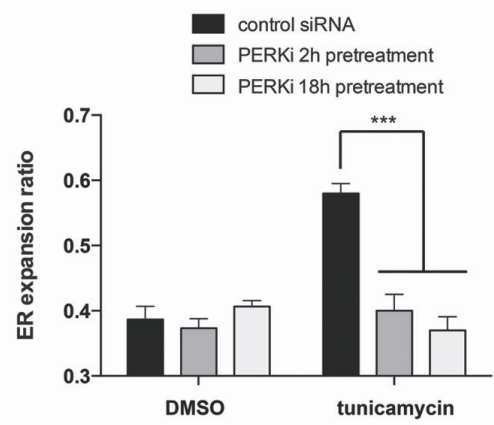

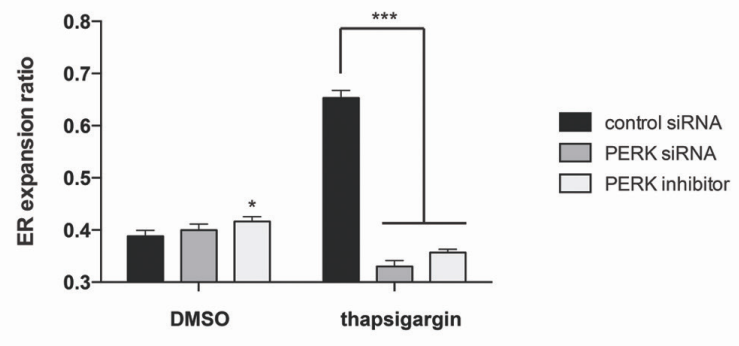

$J$

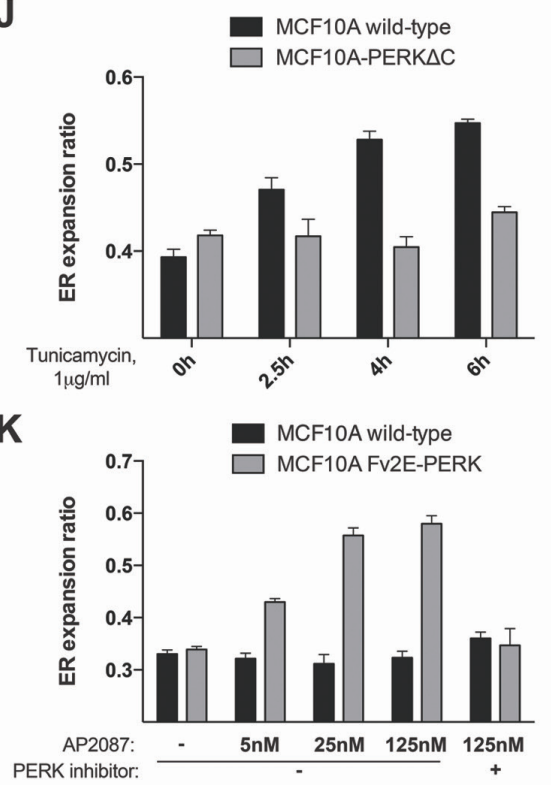

918 Figure 2.- Validation of PERK as an essential effector of acute ER stress-

919 driven ER remodeling. (A, B) Independent analysis of ER remodelling

920 phenotypes for each PERK-targeting siRNA duplex. [A] Representative

921 unprocessed, automatically acquired images of anti-calreticulin- 
922 immunostained MCF10A cells across indicated conditions. [B] Graphs derived

923 from four independent replicates ( 2000 cells per well). (C-E) Allosteric

924 inhibition of PERK kinase activity recapitulates the phenotype observed upon

925 siRNA-mediated PERK depletion. [C] Assessment of the activity and

926 specificity of the inhibitory compound (PERKi; GSK2606414, see Matherials

927 and Methods) by western blot analysis of whole-cell lysates across indicated

928 conditions. [D] Representative images (calreticulin immunostaining) and [E]

929 data derived from eight biological replicates ( 2000 cells per well) across

930 indicated treatment conditions. (F-G) Recapitulation of previous results upon

931 acute ER stress induction using a different mechanism $\left(\mathrm{Ca}^{2+}\right.$ depletion from

932 the ER, using the SERCA2 inhibitor thapsigargin). [F] Representative anti-

933 calreticulin immunofluorescence staining across indicated conditions; [G] data

934 derived from four biological replicates ( 2000 cells per well) across indicated

935 treatments. ( $\mathbf{H}, \mathbf{J})$ Stable expression of an inactive PERK- $\Delta \mathrm{C}$ truncated

936 mutant blunts ER stress-induced PERK signaling and ER peripheral

937 remodelling. Data is derived from eight biological replicates ( 2000 cells per

938 well) across indicated treatments. (I, K) Exposure of cells expressing a

939 synthetic fusion PERK protein with homodimerizing domains (PERK-Fv2E), to

940 the AP20187 homodimerizer enables for PERK kinase-dependent ER

941 remodelling bypassing ER stress induction. Data is derived from eight

942 biological replicates ( 2000 cells per well) across indicated treatments.

943 Statistical significance across assays was assessed by paired t-Student test;

$944 \quad$ *: $p<0.05 ;{ }^{* *}: p<0.01$; ${ }^{* * *}: p<0.005$. n.s.: $p>0.05$ 
A

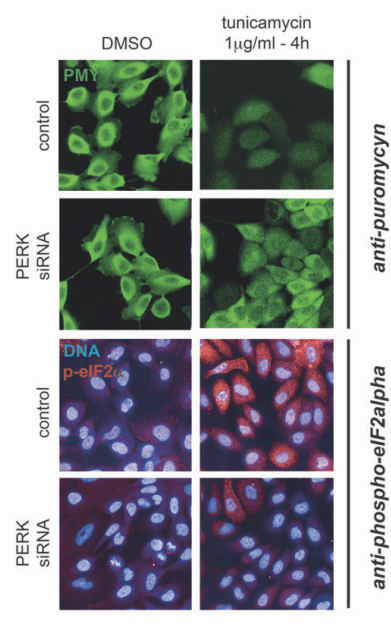

C
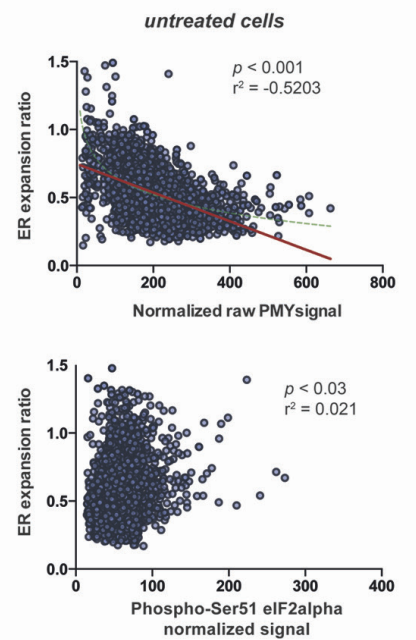

B
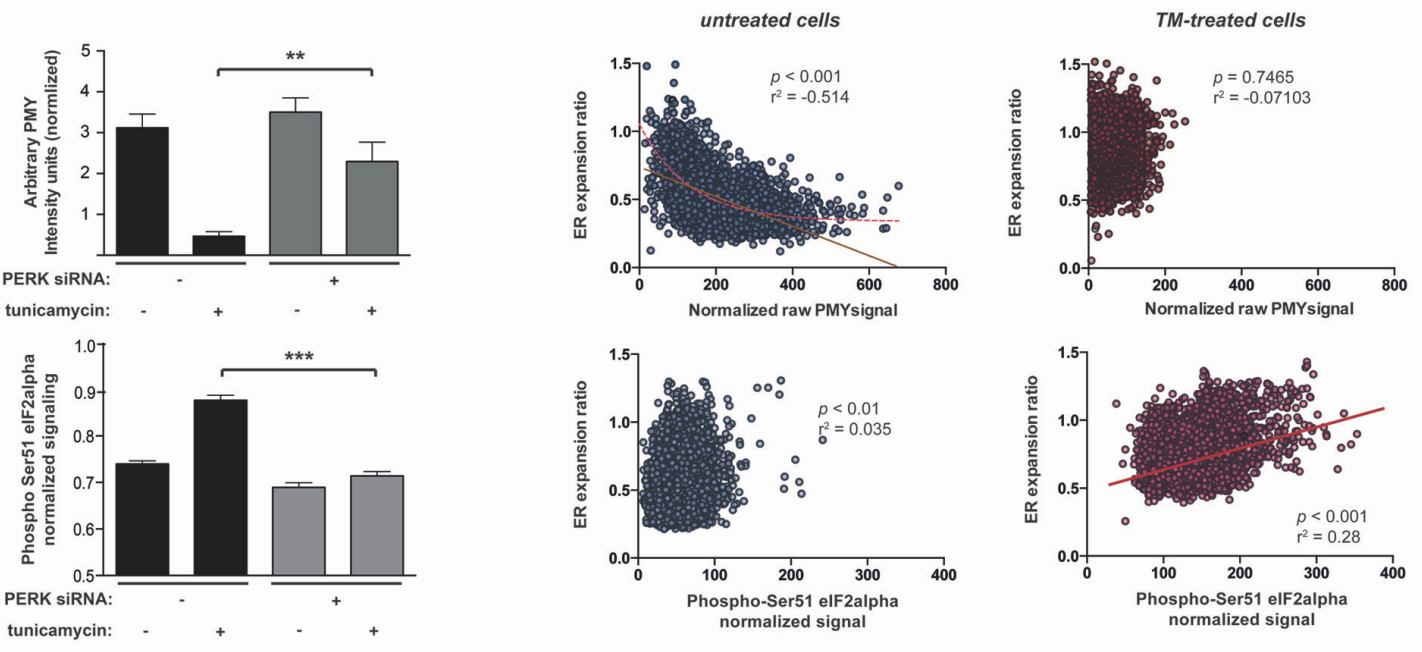

D
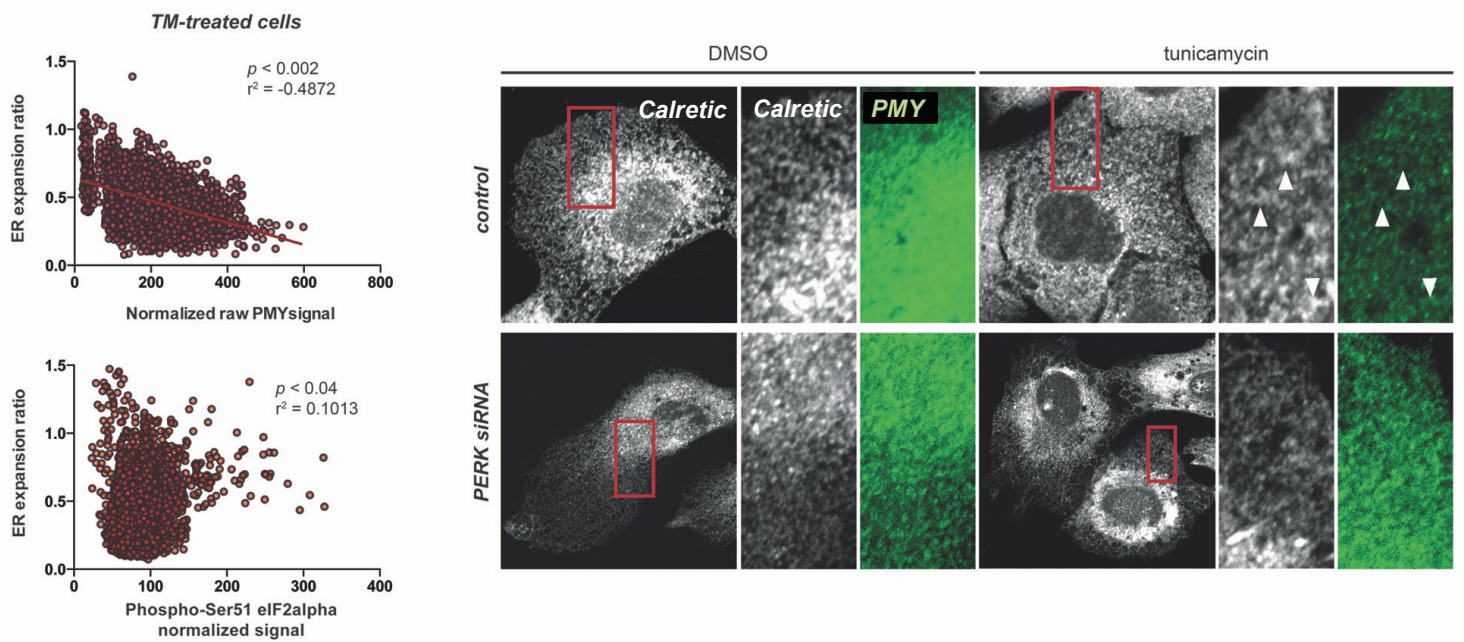

$E$
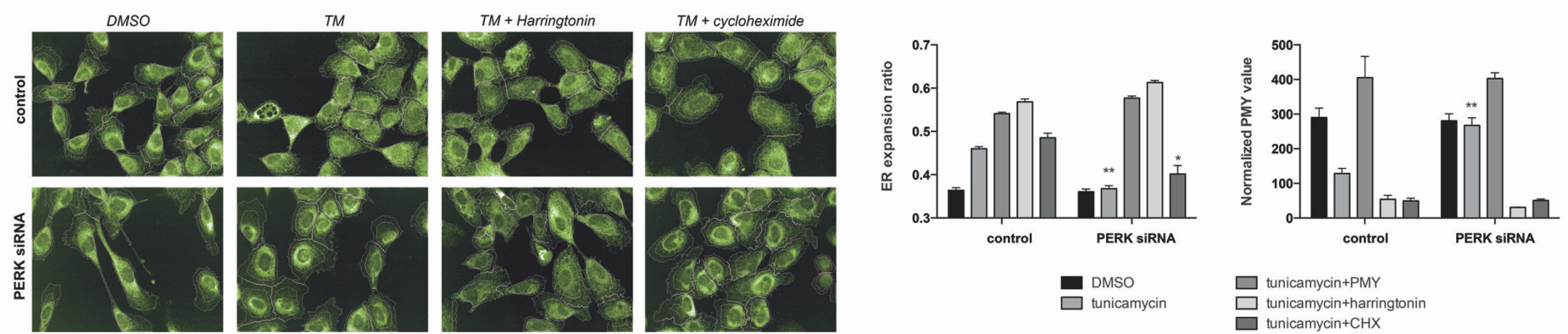

\section{Figure 3.- PERK-dependent translation initiation inhibition correlates}

947 with peripheral ER remodeling. (A) Puromycylation assays reveal a

948 correlation between PERK-dependent protein translation shutdown and elF2a

949 phosphorylation upon exposure to acute ER stress in MCF10A cells. Data are

950 derived from eight biological replicates ( 2000 cells per well) across indicated

951 treatments for each label. (B, C) Plotting of values recorded for single 
952 MCF10A cells from cultures either mock-transfected [B] or PERK-depleted

$953[\mathrm{C}]$. Note correlation and statistical significance of this correlation across 954 treatments between labels, and how this relationship is no longer sensitive to 955 ER stress induction in PERK-depleted cells (D) Single confocal planes of 956 MCF10A cells double-stained for ER (calreticulin, white) and active translation 957 (PMY, green) across indicated siRNA and compound treatments. Note that 958 peripheral sheet-like patches in mock-transfected cells exposed to ER stress 959 are particularly devoid of PMY label (arrowheads). (E) Pharmacological 960 inhibition of protein translation initiation, but not translation elongation, reverts 961 the impairment for peripheral ER remodeling in PERK-depleted cells. PMY 962 stands for both puromycylation label, and sustained inhibitory treatment. CHX: 963 cycloheximide. Data is derived from four biological replicates ( 2000 cells per 964 well) across indicated treatments. Statistical significance across assays was 965 assessed by paired t-Student test; *: $p<0.05 ;{ }^{* *}: p<0.01$; ${ }^{* *}: p<0.005$. n.s.: $p$ $966>0.05$ 
A

B

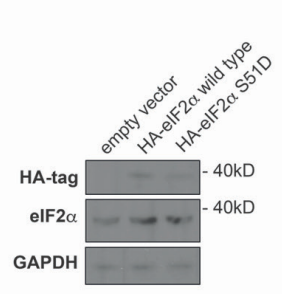

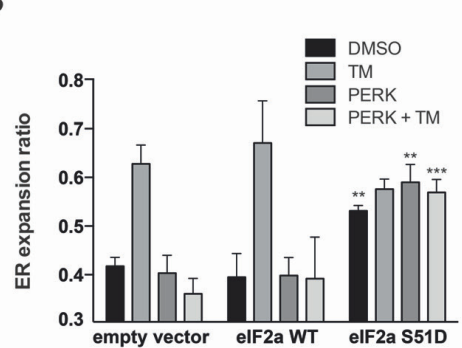

C

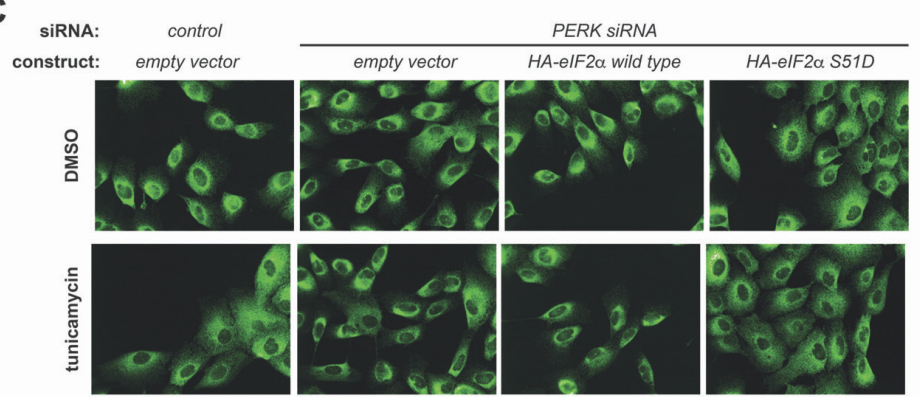

D

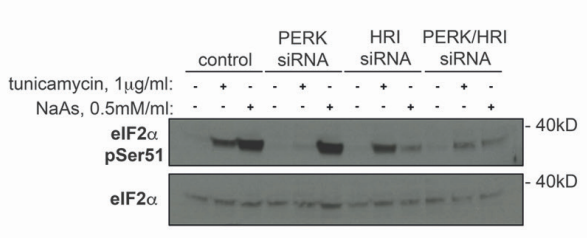

E
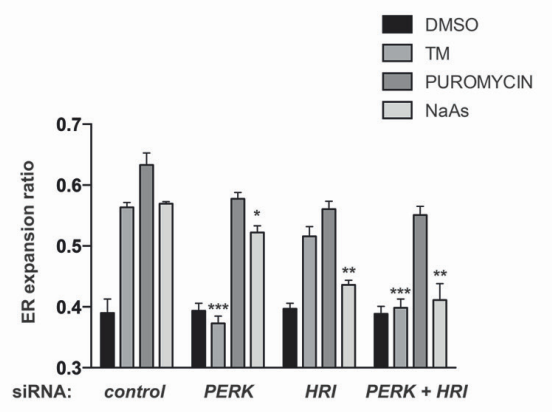

$\mathbf{F}$

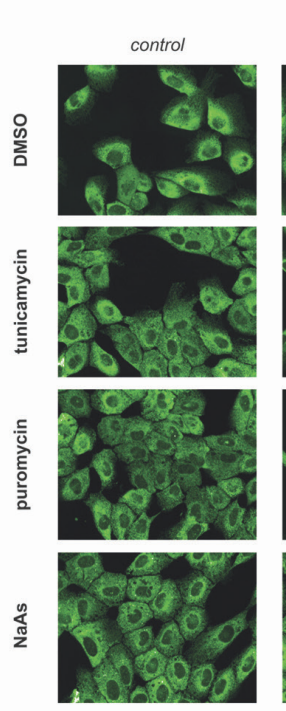

PERK SIRNA
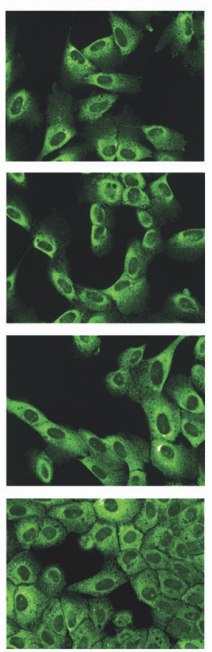

HRI SIRNA
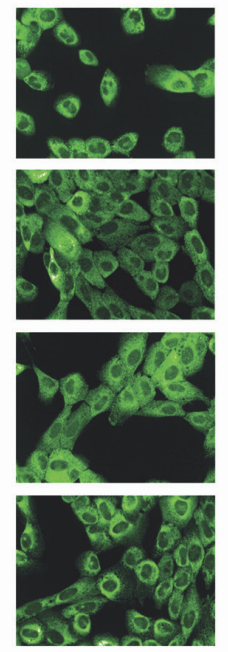

PERK SIRNA HRI SIRNA
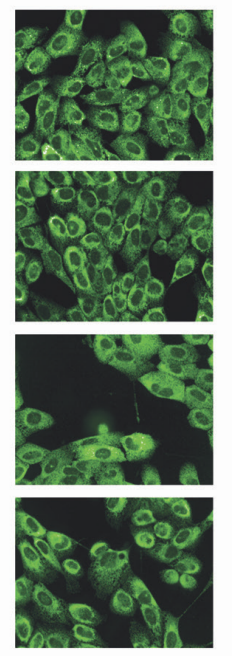

G

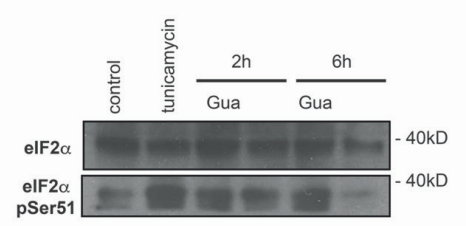

H

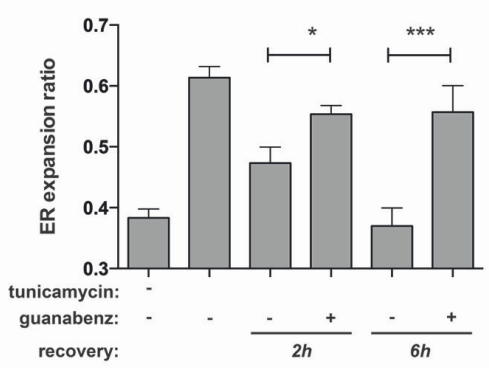

I

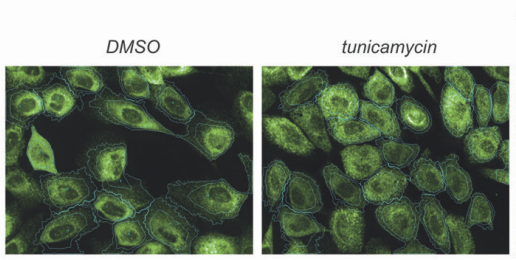

guanabenz, $2.5 \mathrm{nM}$

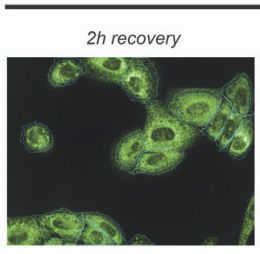

$6 h$ recovery

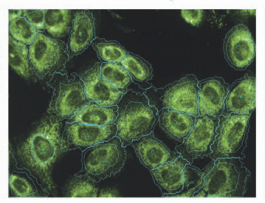

DMSO recovery

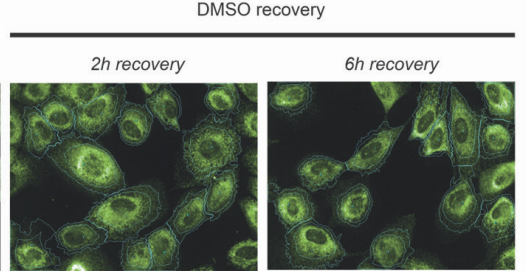

guanabenz, 10nM

$2 h$ recovery

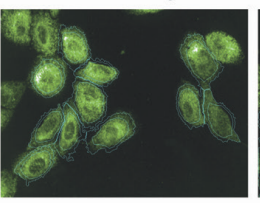

6h recovery

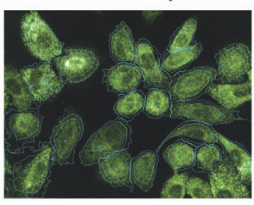

968 Figure 4.- elF2a phosphorylation is required for PERK-dependent

969 induction of peripheral ER remodeling during ER stress. (A-C) Cells

970 stably expressing a phosphomimicking elF2a-S51D or the wild type

971 counterpart [A, westernblot analysis] were assessed for peripheral ER

972 remodelling across indicated conditions $[B, C]$. Data is derived from four 
973 biological replicates ( 2000 cells per well). (D-F) Cells transfected with 974 indicated siRNA duplexes were exposed to indicated treatments, and 975 analyzed for elF2a phosphorylation [D] or peripheral ER expansion [E, F]. 976 Data is derived from four biological replicates ( 2000 cells per well). (G-I) 977 MCF10A cells were exposed to tunicamycin for $4 \mathrm{~h}$, and then fixed, are 978 allowed to recover for indicated times in the presence or absence of 979 guanabenz. Whole-cell extracts were subjected to western blot analysis for 980 indicated markers [G]; fixed samples were processed for immunostaining for 981 calreticulin and analyzed for relative peripheral ER content. Data is derived 982 from four biological replicates ( 2000 cells per well). Statistical significance 983 across assays was assessed by paired t-Student test; *: $p<0.05 ;{ }^{* *}: p<0.01$; $984^{* * *}: p<0.005$. n.s.: $p>0.05$ 
A
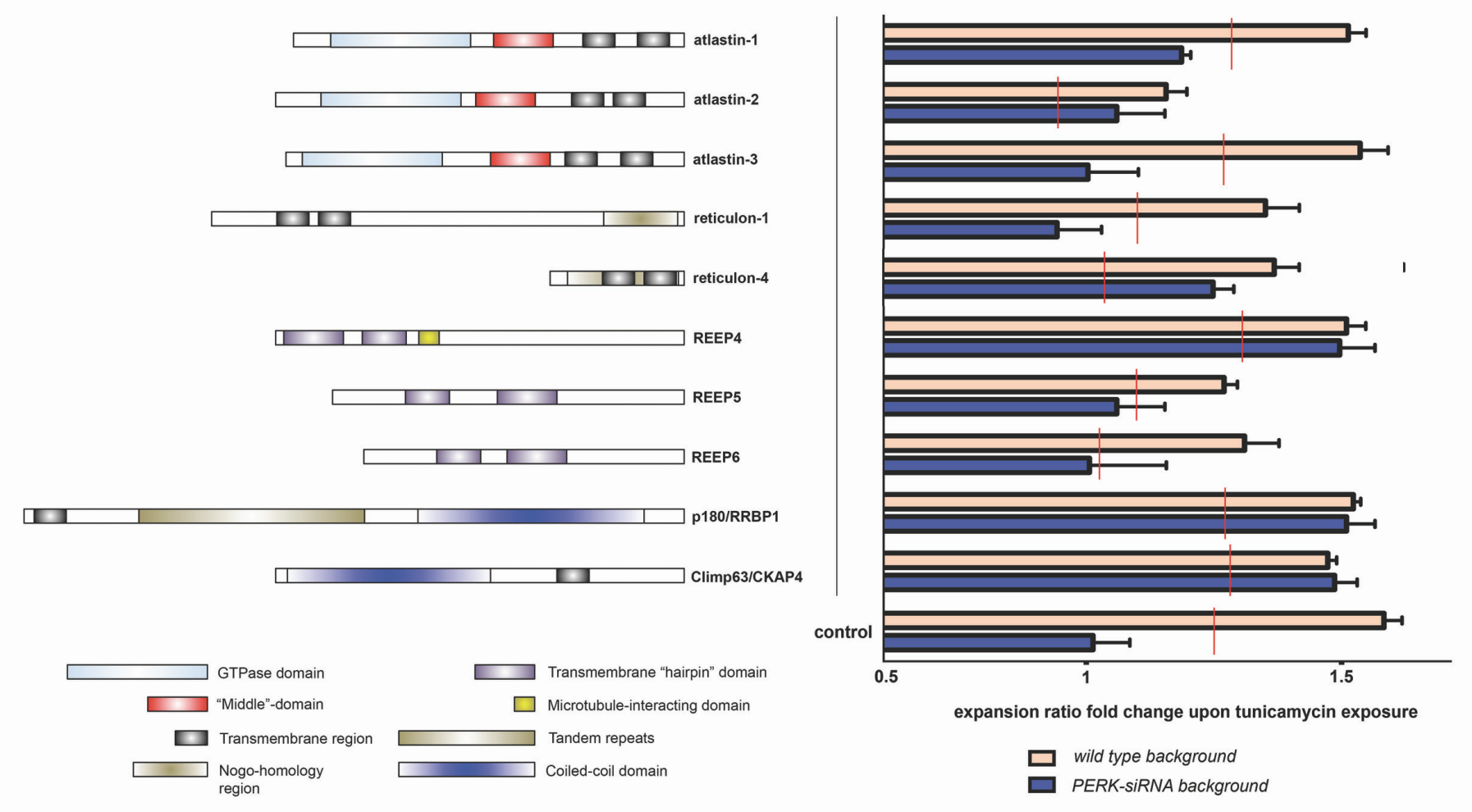

B

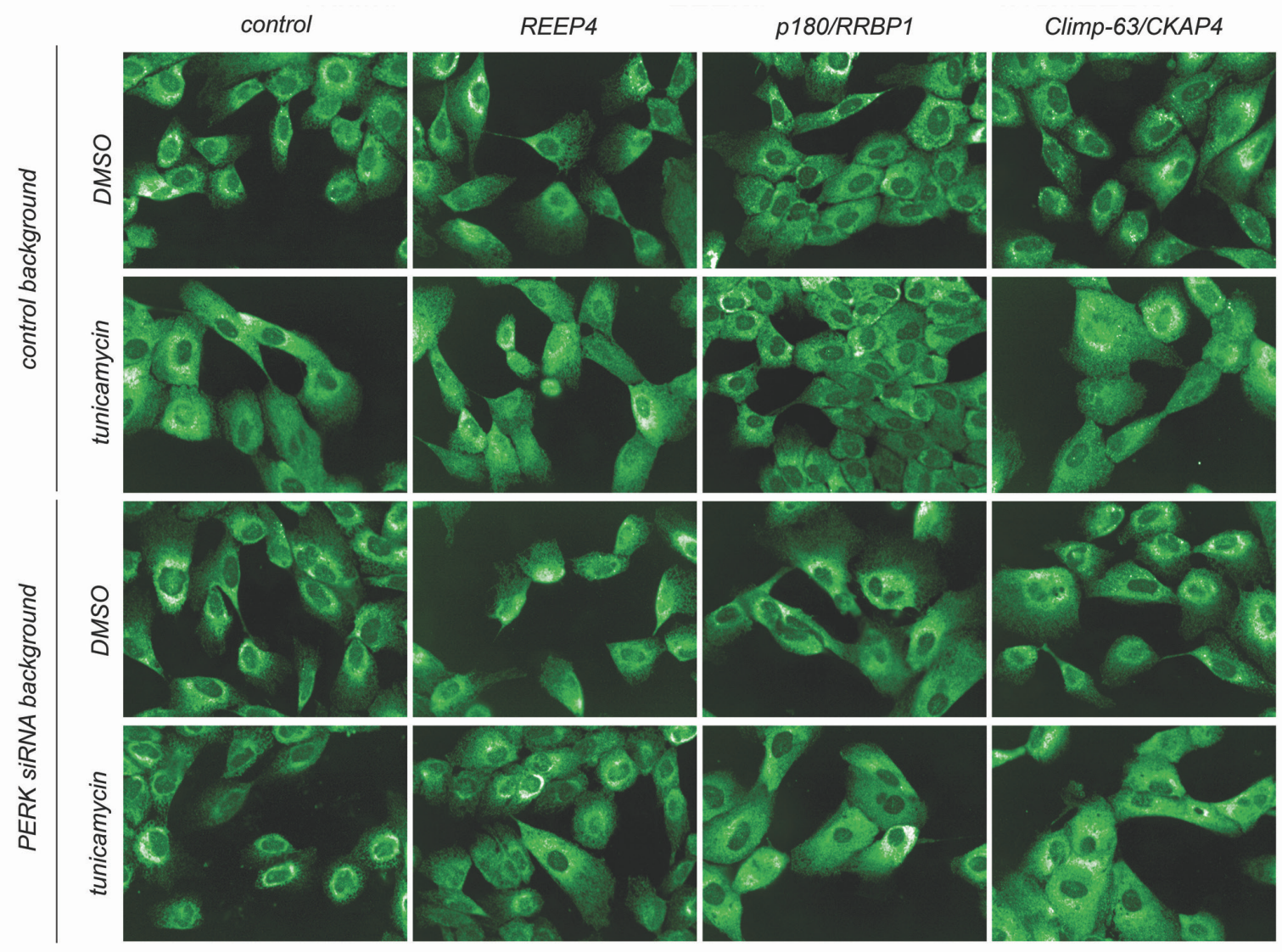

986 Figure 5.- Impaired peripheral ER remodeling in PERK-depleted cells is 
987 reverted upon depleting specific ER 'shaping' proteins that link the ER

988 to the microtubule cytoskeleton. (A) The indicated ER shapers were 989 targeted for transient depletion in MCF10A cells by transfecting siRNA pools, 990 either alone or in combination with siRNA duplex targeting PERK. 48h later, 991 cell subsets were mock-treated with vehicle (DMSO) or exposed for 6h to 992 tunicamycin, and samples were fixed and processed for calreticulin 993 immunofluorescent staining and automated microscopy. Increase in 'ER 994 expansion ratio' in tunicamycin-treated subsets, as compared to their DMSO995 treated counterparts, is shown for each siRNA group. Red lines indicate the 996 threshold of significance $(p<0.05)$ for the difference between single siRNA 997 treatment and the PERK-ER shaper double siRNA combination. Highlighted 998 targets exhibit behavior comparable to mock-silenced cells, and rescue of the 999 phenotype associated with PERK depletion. Data were derived from four 1000 biological replicates ( 2000 cells per well). (B) Representative images of 1001 indicated siRNA combinations. Statistical significance across assays was 1002 assessed by paired t-Student test; ${ }^{*}: p<0.05 ;{ }^{* *}: p<0.01$; ${ }^{* * *}: p<0.005$. n.s.: $p$ $1003>0.05$ 
A
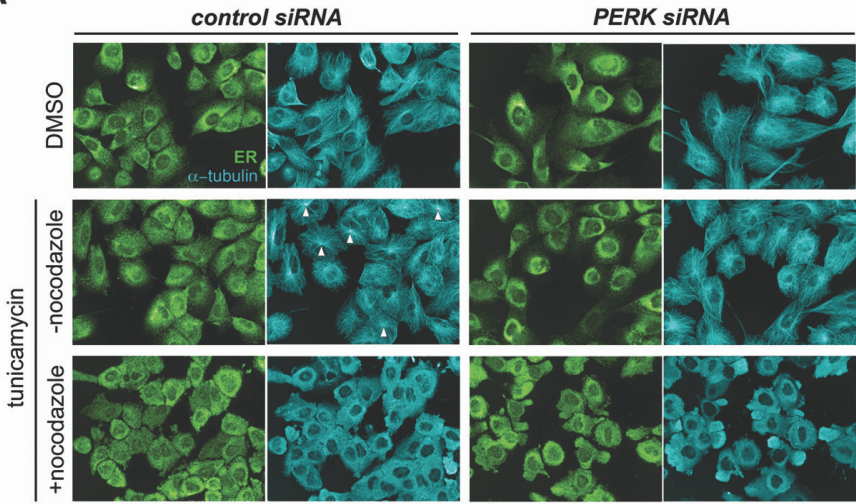

C

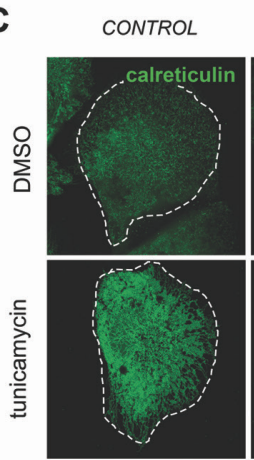

PERK SIRNA

CAMSAP2 SIRNA

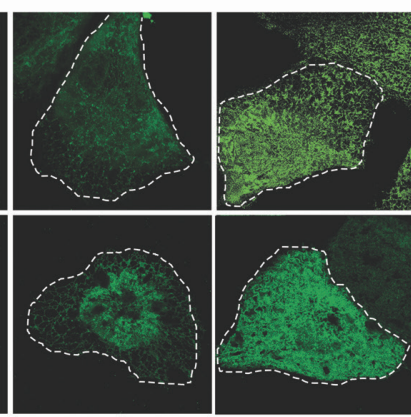

D

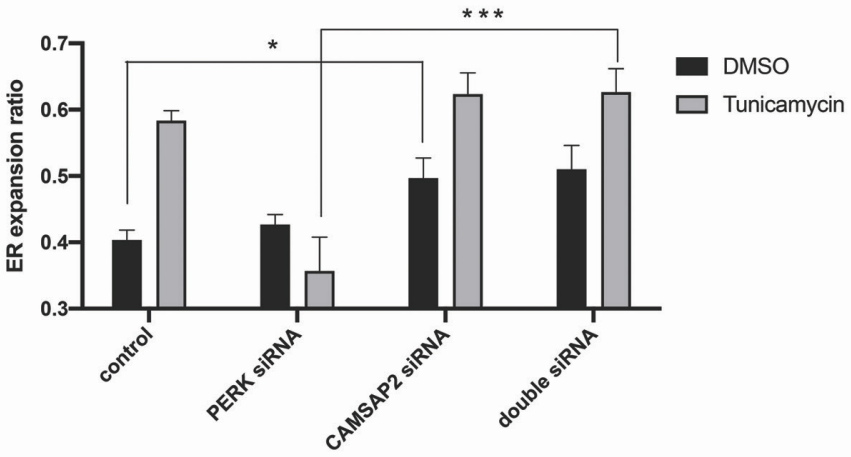

E
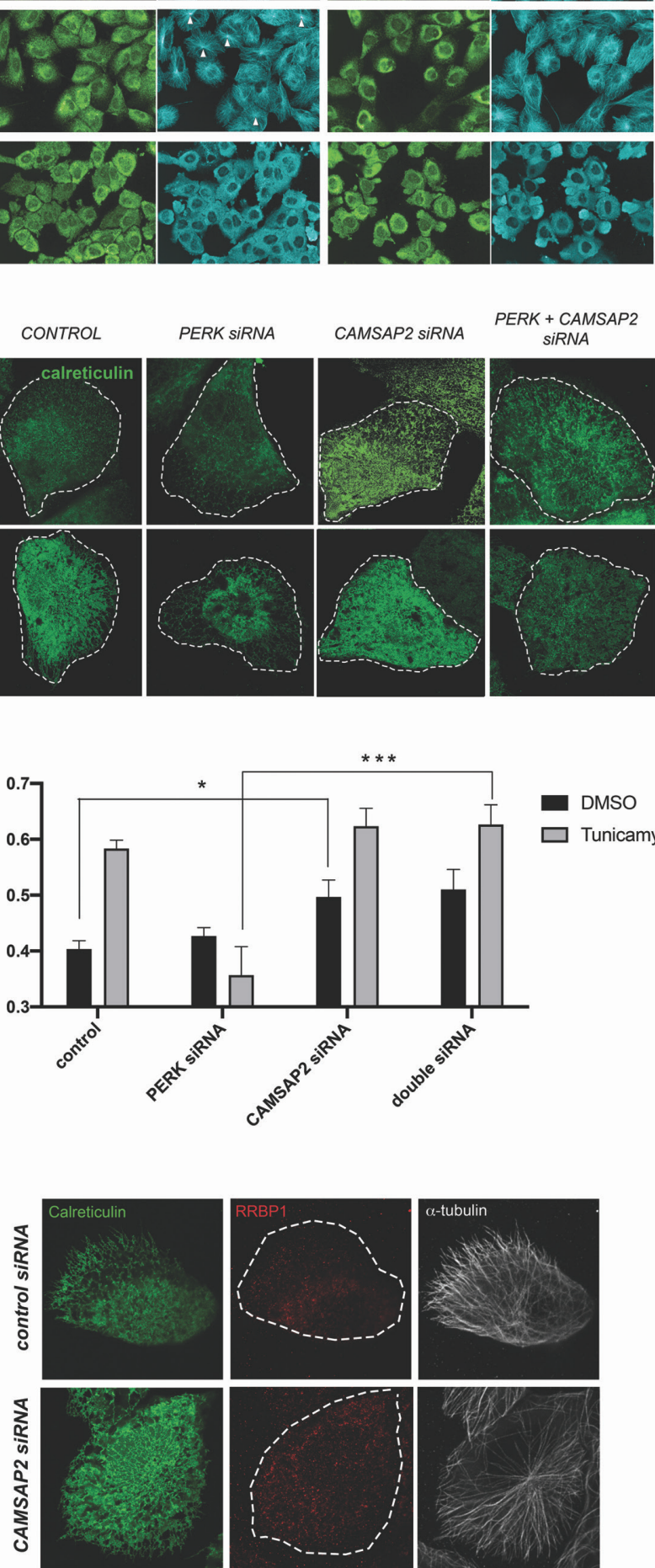

PERK + CAMSAP2

$\mathbf{F}$
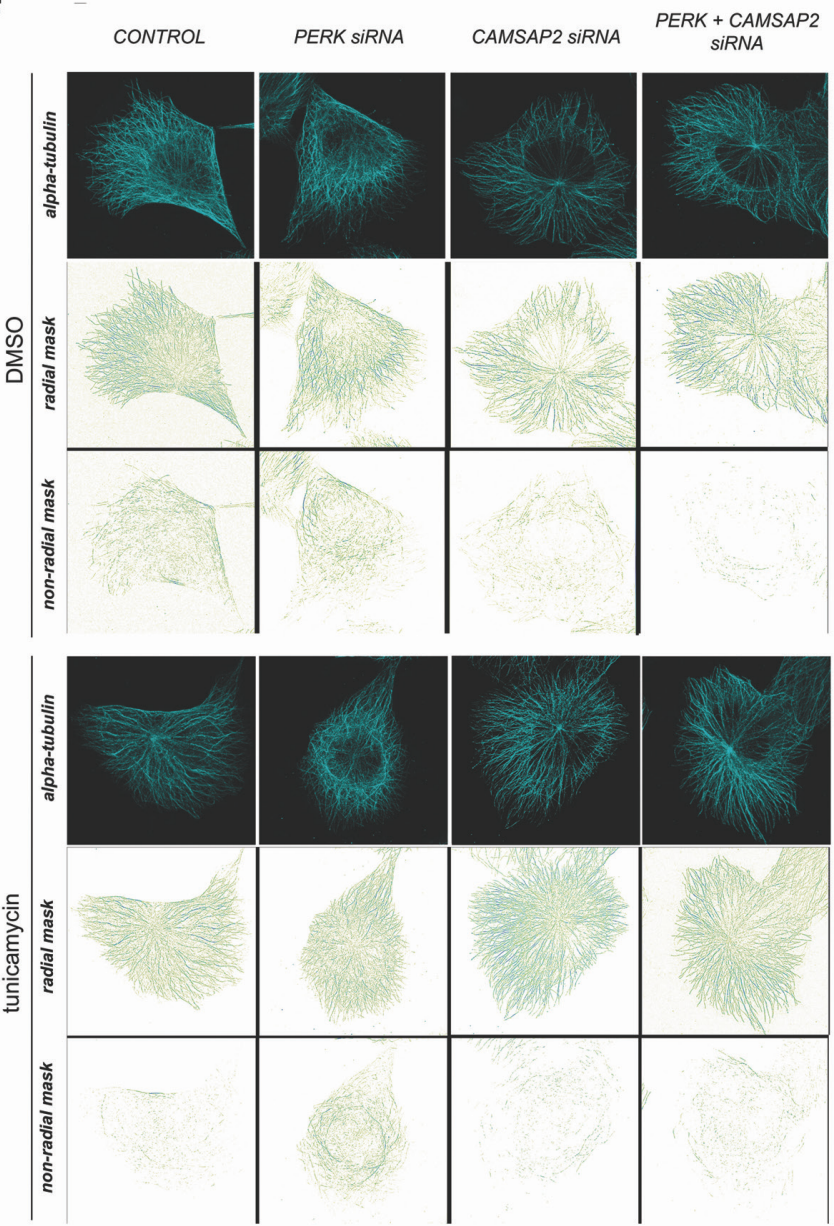

G

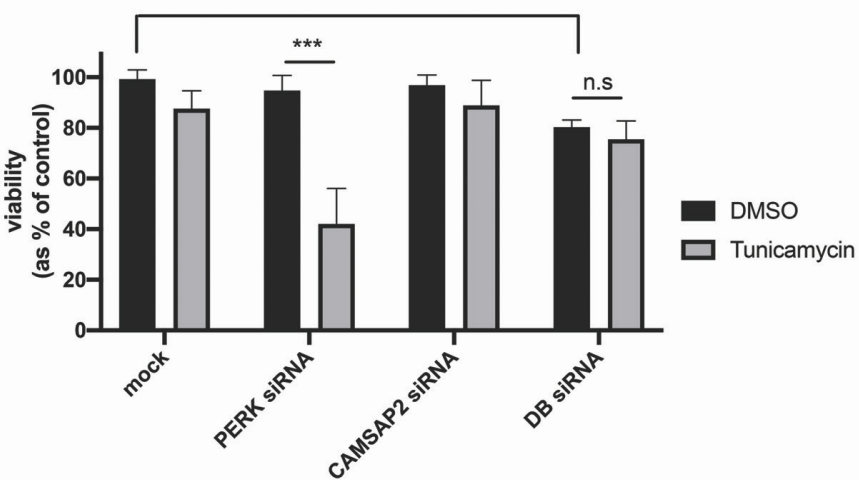

1005 Figure 6.- Non-centrosomal microtubules are specifically required for

1006 PERK depletion to induce a phenotype of impaired peripheral ER

1007 remodeling. (A) MCF10A cells were subjected to indicated siRNA and small

1008 compound treatments, fixed and immunostained for ER and microtubules, and 
1009 imaged. Arrowheads indicate cells with clear radial disposition of 1010 microtubules. (B-D) MCF10As were reverse-transfected with indicated siRNA 1011 combinations and exposed to indicated small-compound treatments, and 1012 analyzed by western blot [B] or quantitative imaging [C, D]. Data in [D] were 1013 derived from four biological replicates ( 2000 cells per well). (E) Triple 1014 immunostaining for endogenous calreticulin (green), RRBP/p180 (red) and $\alpha$ 1015 tubulin (grayscale) in wild type MCF10A cells and cells depleted for 1016 CAMSAP2; cell boundary is highlighted with dashed lines. (F) Analysis of 1017 radial and non-radial microtubule signals from MCF10A cells subjected to 1018 indicated siRNA and small-compound treatments. (G) MTT viability assay 1019 across indicated conditions. Data were derived from eight biological 1020 replicates. Statistical significance across assays was assessed by paired t1021 Student test; *: $p<0.05 ;{ }^{* *}: p<0.01$; ${ }^{* *}: p<0.005$. n.s.: $p>0.05$ 
A

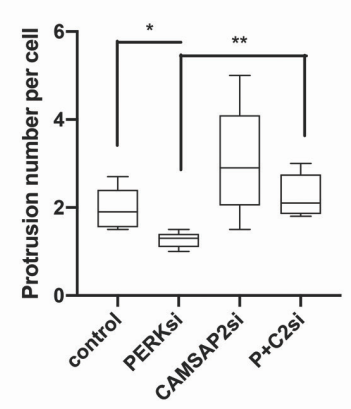

D

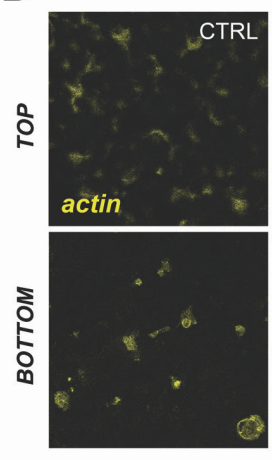

$\mathbf{F}$

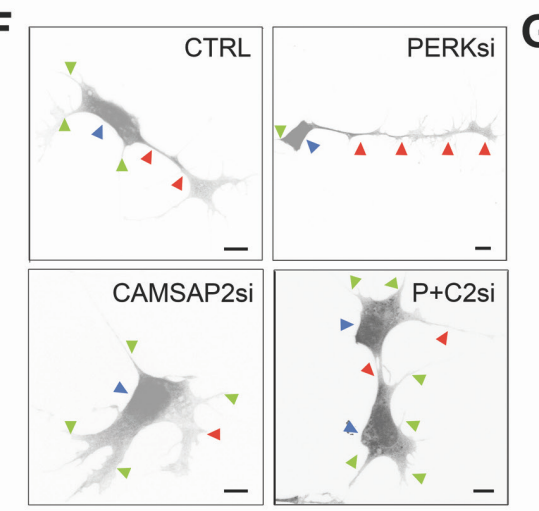

B

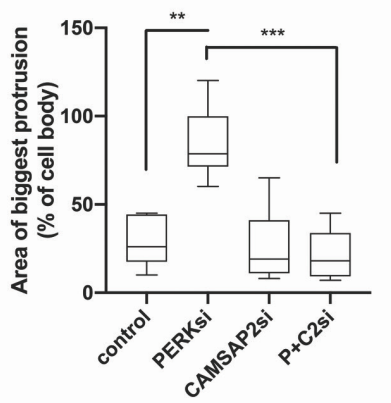

C

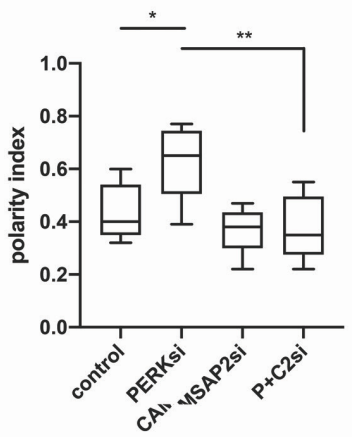

E

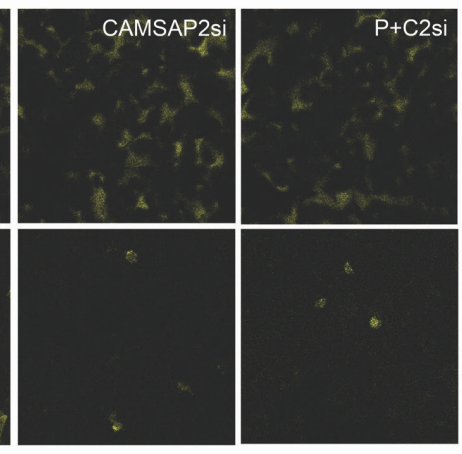

G

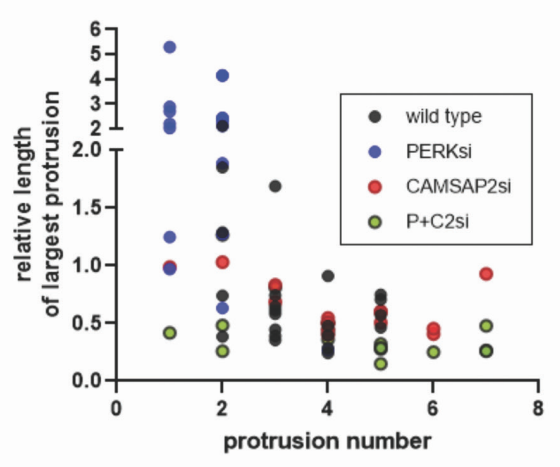

H

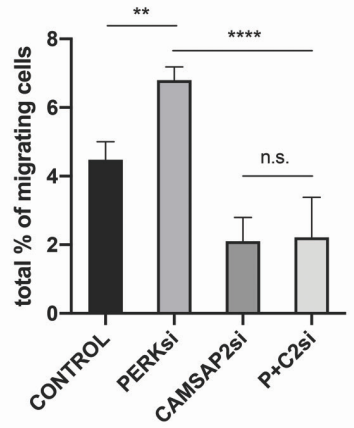

1023 Figure 7.- PERK depletion impacts on cell protrusiveness and polarized

1024 migration in a manner dependent on non-centrosomal microtubules. (A-

1025 C) Features indicated were extracted from a minimum 25 microtubule- and

1026 Golgi immunostained, cytoplasm-counterstained cell confocal images across

1027 indicated conditions. (D-E) MCF10As subjected to indicated siRNA treatments

1028 were allowed to protrude through Transwell ${ }^{\mathrm{TM}}$ pore membranes for $4 \mathrm{~h}$, then

1029 fixed and stained for actin and imaged. [E] Indicated parameters were

1030 computed from 10 independent fields per condition, from 3 biological

1031 replicates. (F-G) SH-Sy5y neuroblasts transfected with indicated esiRNAs

1032 were stimulated for neural differentiation (see M\&Ms). Cell soma (blue 1033 arrowheads), the longest protrusion (red arrowheads) and secondary 
1034 protrusions (green arrowheads) are highlighted. Scale bar: 50um [G] The 1035 number of protrusions per cell, and the length of the longest protrusion 1036 relative to the perimeter of the cell soma are plotted for each condition $(n=20$ 1037 from 2 biological replicates). (H) MCF10As subjected to indicated siRNA 1038 treatments were allowed to migrate through 3D collagen matrices, and 1039 imaged at plate bottom, and three 50micron-apart optical sections. \% of cells 1040 migrated from total cell count is indicated. Data were derived from six 1041 biological replicates. Statistical significance across assays was assessed by 1042 paired t-Student test; *: $p<0.05$; ${ }^{* *}: p<0.01$; ${ }^{* * *}: p<0.005$. n.s.: $p>0.05$. Error 1043 bars depict S.E.M. 


\section{SUPPLEMENTARY FIGURES:}

A

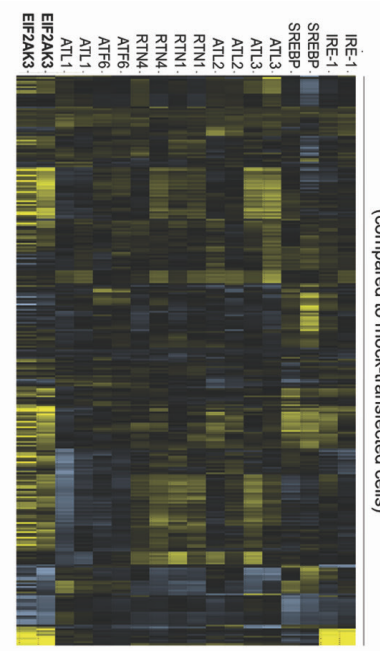

D

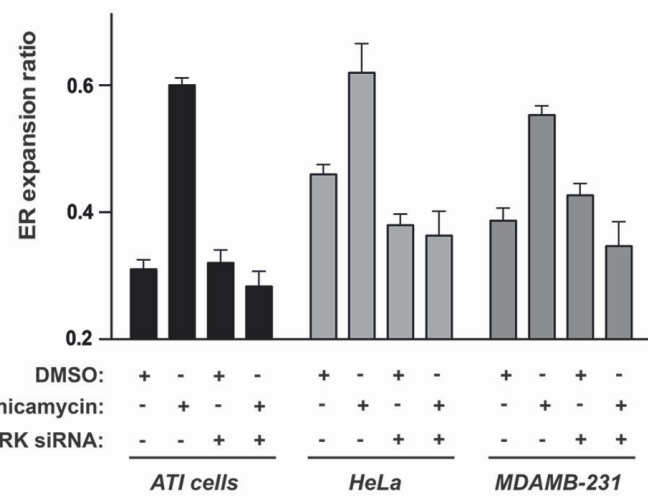

E

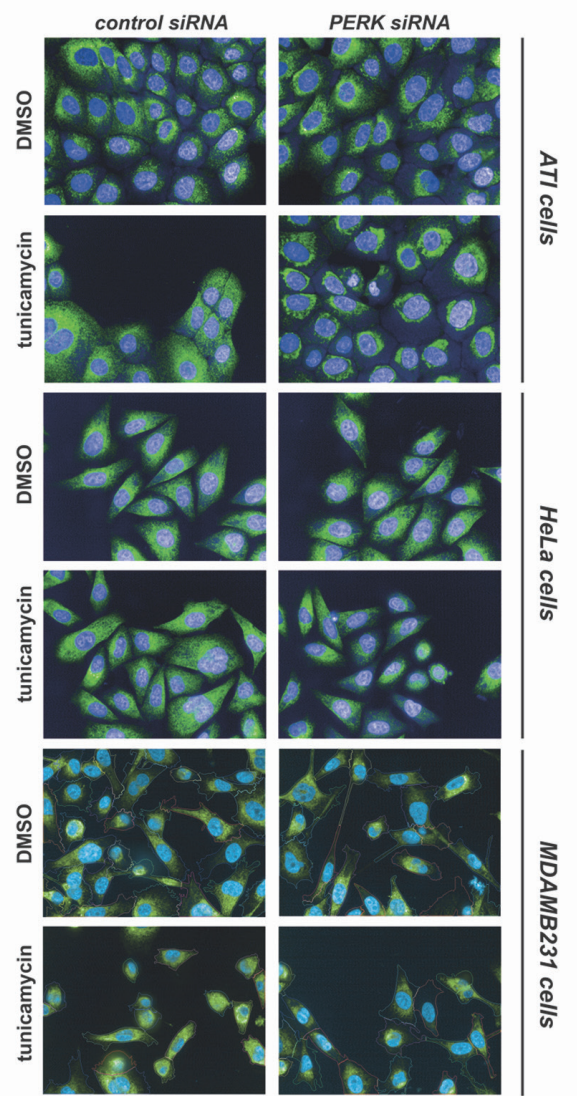

B
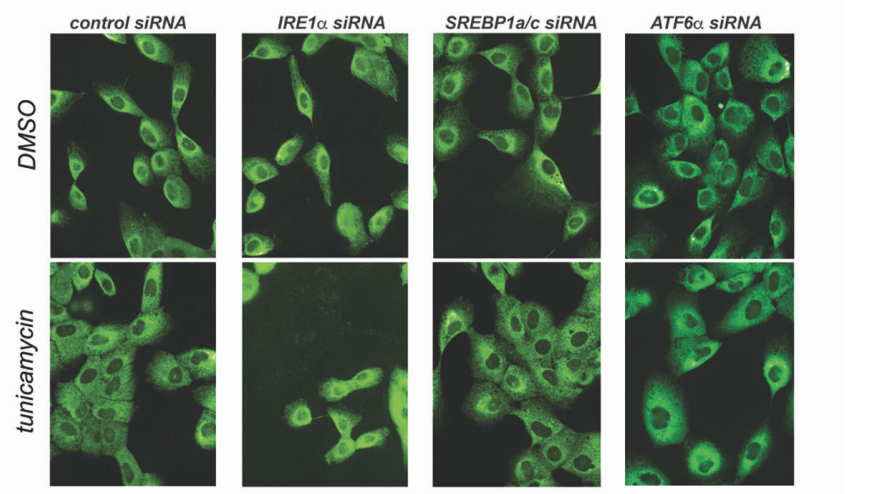

C

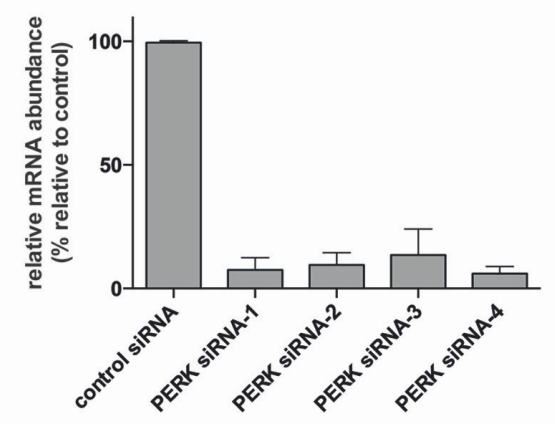

$\mathbf{F}$

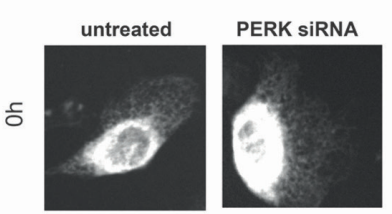

PERKi
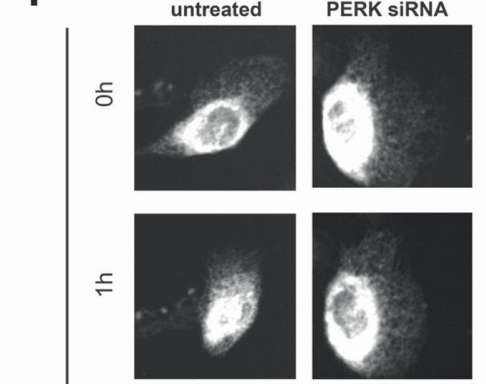

(2h pretreatment)

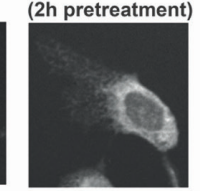

단
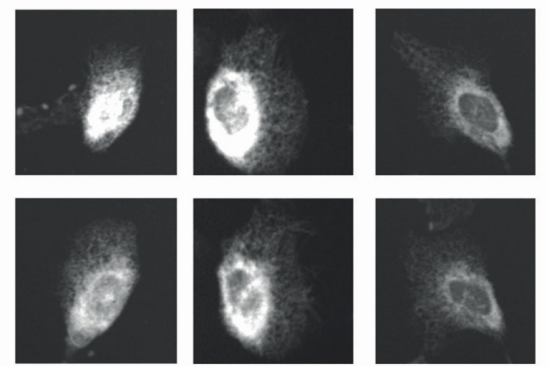

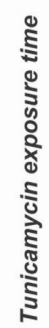

달
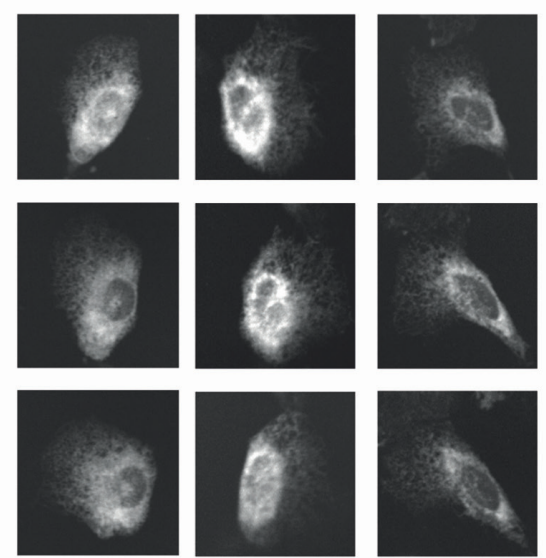

ᄃำ
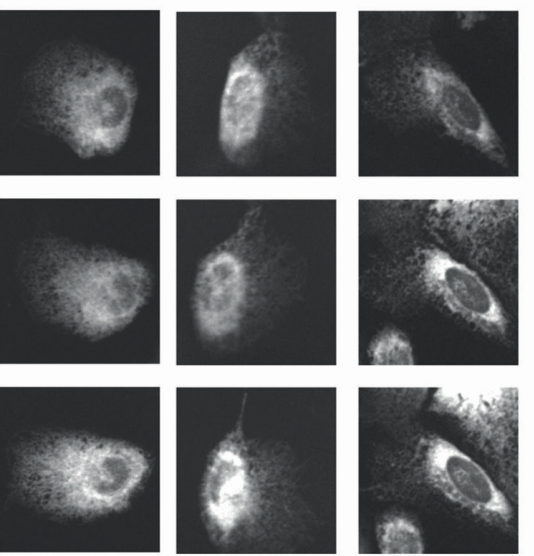
1046 Supplementary Figure 1.- (A) Heatmap showcasing Z-scores extracted from

10472 biological replicates (averaged for 4 replicate wells for each condition) of all

1048136 image features (both including treated and non-treated conditions) across

1049 indicated siRNA pairs. Hierarchical clustering renders correct pairing of siRNA

1050 duplex replicas. (B) Immunofluorescence images (calreticulin) of cells

1051 transfected with indicated siRNA duplexes and exposed to either vehicle

1052 (DMSO) or $1 \mu \mathrm{g} / \mathrm{ml}$ tunicamycin for $6 \mathrm{~h}$. Note impaired remodelling in IRE1 and

1053 SREBP1a/c-depleted cells. (C) RT-PCR analysis of EIF2AK3 mRNA levels in

1054 cells transfected with indicated siRNA duplexes (related to figure 2A). Data is

1055 derived from 3 technical replicates (D, E) Indicated cell lines were reverse

1056 transfected with an siRNA pool targeting PERK or scrambled, subjected to

1057 indicated treatments and processed for immunofuorescent staining

1058 (calreticulin; counterstained for DAPI), imaged and analysed. Graphs are

1059 derived from four independent replicates ( 2000 cells per well). (F) Live cell

1060 imaging of MCF10A cells stably expressing an EGFP-Sec61 $\beta$ fusion, for

1061 indicated times and across indicated treatments. Statistical significance

1062 across assays was assessed by paired t-Student test; ${ }^{*}: p<0.05$; ${ }^{* *}: p<0.01$;

$10633^{* * *}: p<0.005$. n.s.: $p>0.05$ 
A

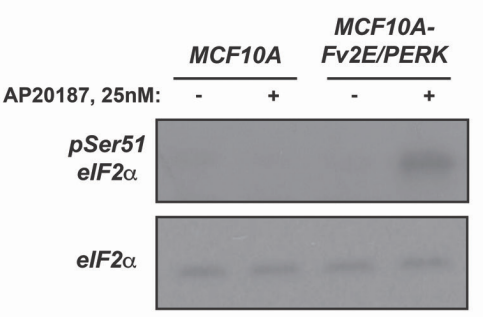

C

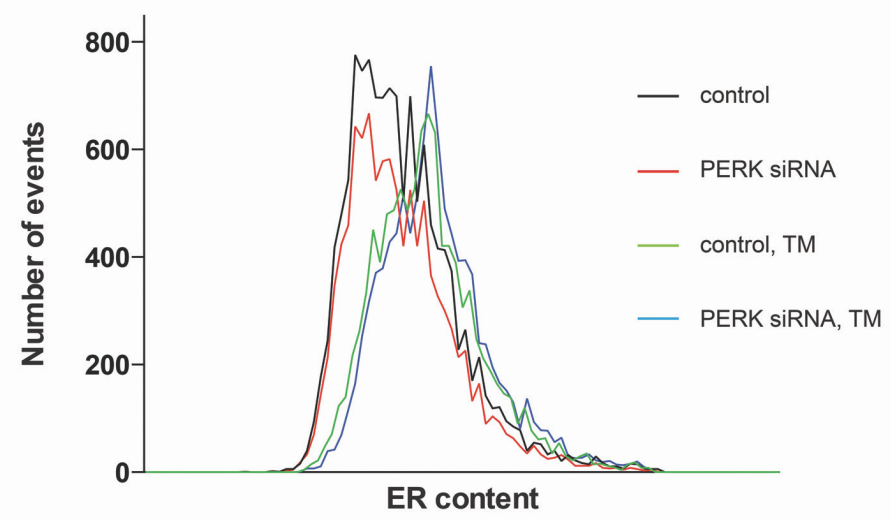

$\mathbf{E}$
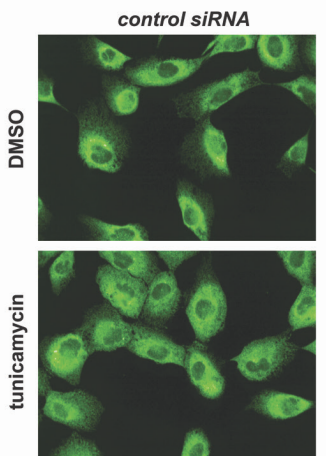

B

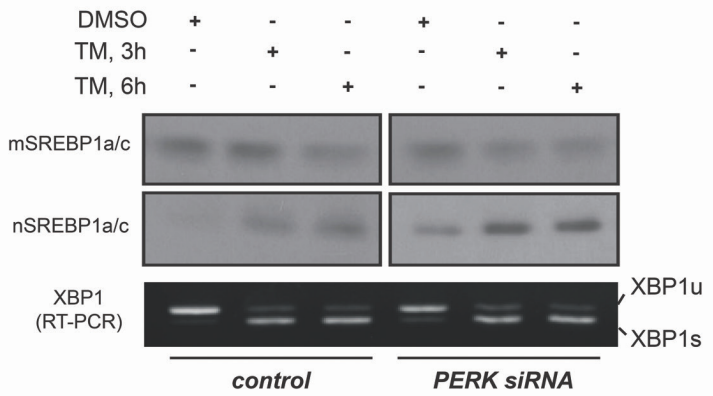

D

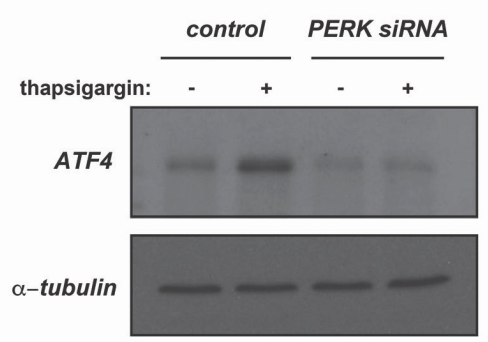

$\mathbf{F}$

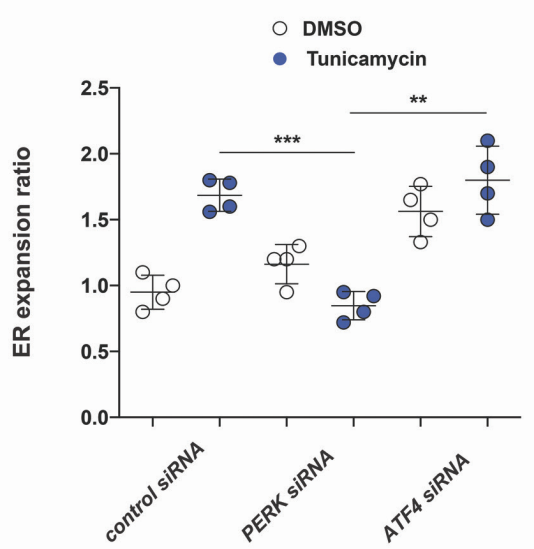

1065 Supplementary Figure 2.- (A) Whole-cell extracts from MCF10A or MCF10A-

1066 Fv2E-PERK cells treated as indicated, were analyzed by western blotting with

1067 indicated primary antibodies. (B) Protein and total RNA was extracted from

1068 cells treated as indicated and analyzed by western blot and RT-PCR. (C)

1069 Cells treated as indicated were detached and labeled with ER Tracker-

1070 BODIPY FL for 15min, and analyzed by flow cytometry for ER total content.

1071 (D) Whole-cell extracts from MCF10A cells treated as indicated, were

1072 analyzed by western blotting with indicated primary antibodies. (E, F)

1073 MCF10A cells were reverse transfected with an siRNA pool targeting ATF4 or

1074 scrambled, subjected to indicated treatments and processed for

1075 immunofuorescent staining (calreticulin; counterstained for DAPI), imaged and

1076 analysed. Graphs are derived from four independent replicates ( 2000 cells 
1077 per well). Statistical significance across assays was assessed by paired t-

1078 Student test; ${ }^{*}: p<0.05 ;{ }^{* *}: p<0.01 ;{ }^{* *}: p<0.005$. n.s.: $p>0.05$ 
A

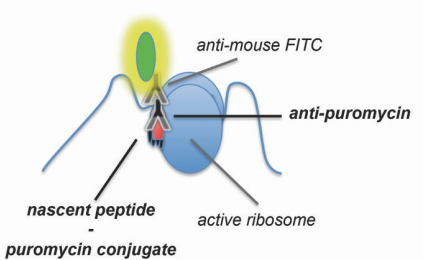

C
B

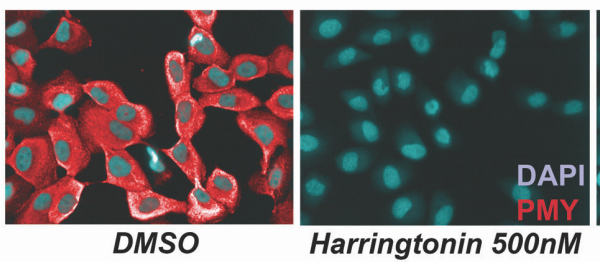

45", 300ng/ml puromycilation pulse

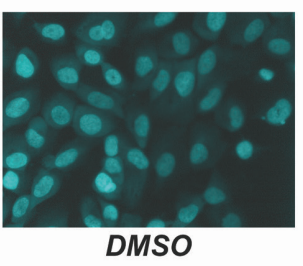

mock pulse

MCF10A-wild type
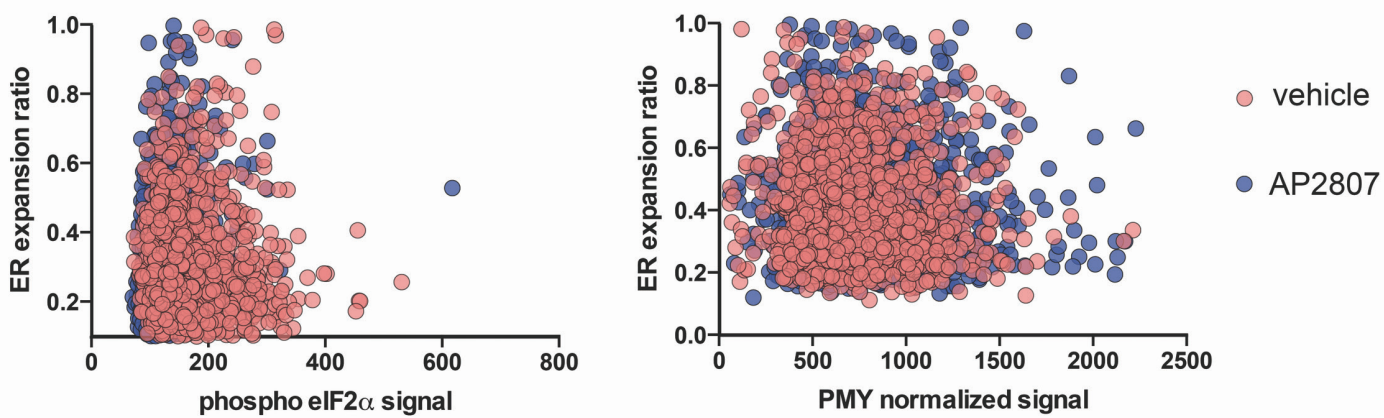

MCF10A-Fv2E-PERK
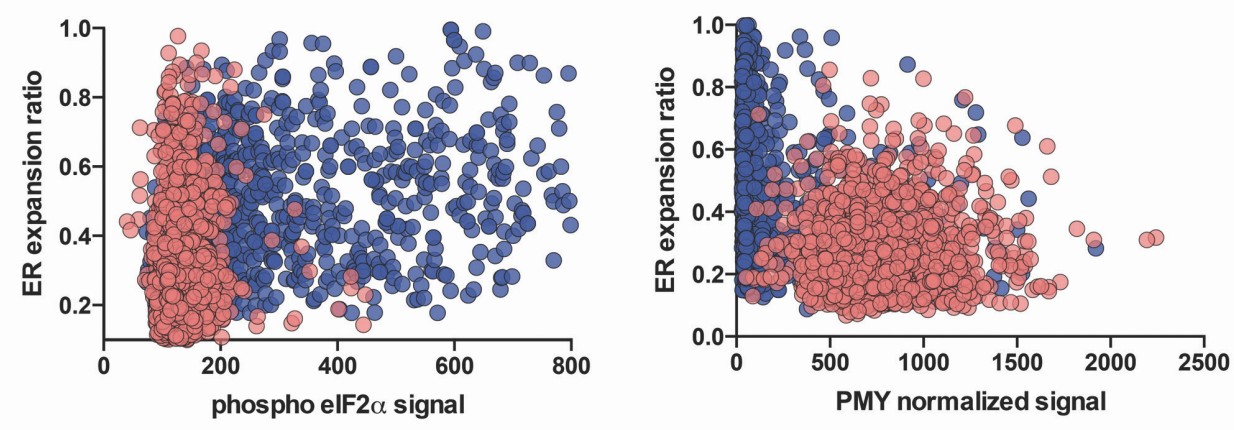

vehicle

- AP2807

1080 Supplementary Figure 3.- (A) Diagram of puromycylation immunostaining

1081 technique (B) Control assay showing specificity of the technique. (C) The 1082 synthetic Fv2E-PERK homodimerization system bypassing ER stress 1083 activation recapitulates the observations on ER expansion correlation and 1084 translation shutdown at single-cell level (related to figure 3B and C). 
A

B
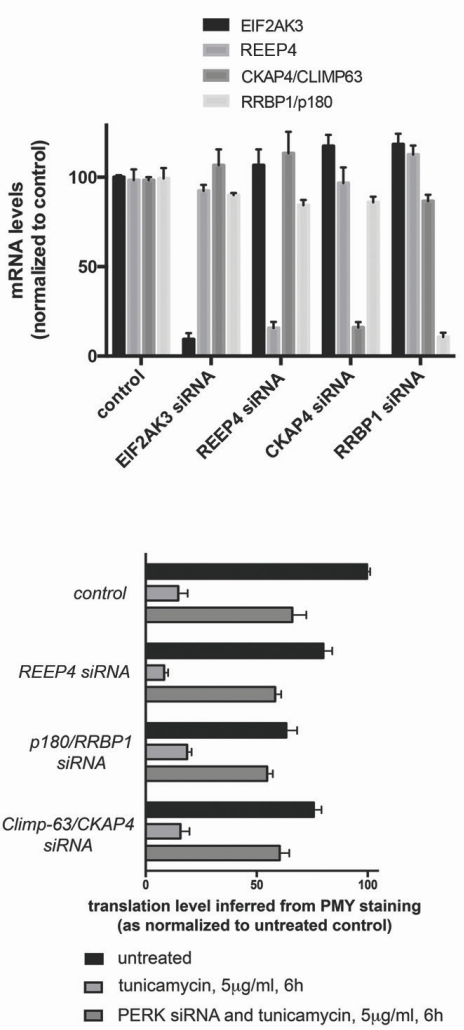

C

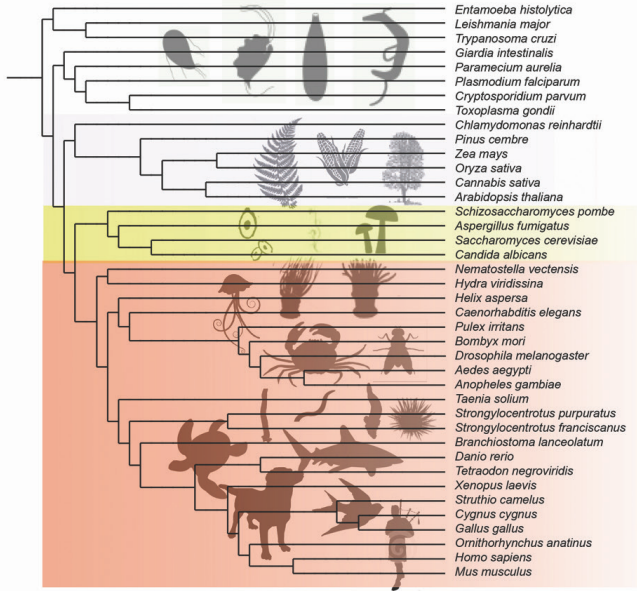

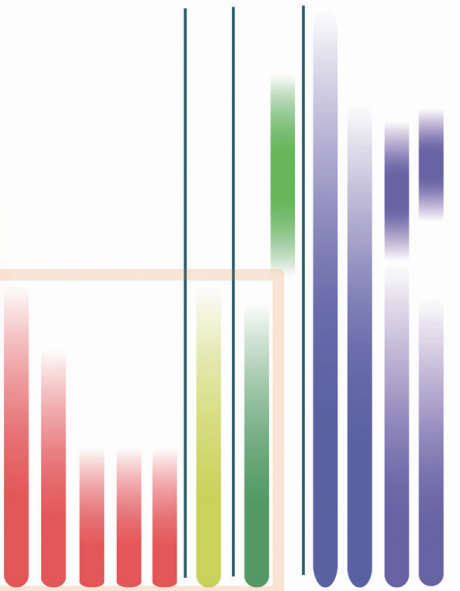

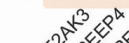

1086 Supplementary Figure 4.- (A) qRT-PCR analysis of indicated mRNA

1087 transcripts across indicated conditions. Data are derived from 3 biological

1088 replicates. (B) Image-based puromycylation assay across indicated conditions

1089 (4 biological replicates, approx. 2000 cells each). Data are expressed as

1090 normalized to control untreated condition, which is set as $100 \%$. (C) Visual

1091 rendering of estimated evolutionary conservation of indicated genes, as

1092 related to specific features of ER-cytoskeleton relationship. Evolutionary

1093 conservation is derived from InParanoid database, and layered over an

1094 evolutionary tree across indicated eukaryotic phila (OrthoDB, University of

1095 Geneva). 
A

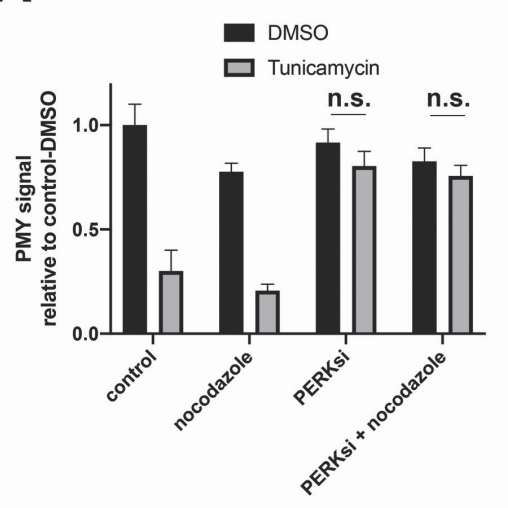

C

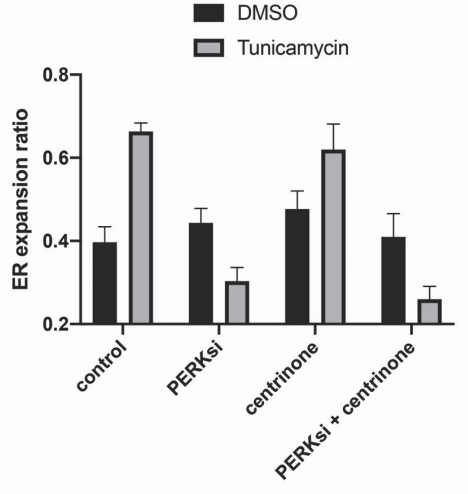

B
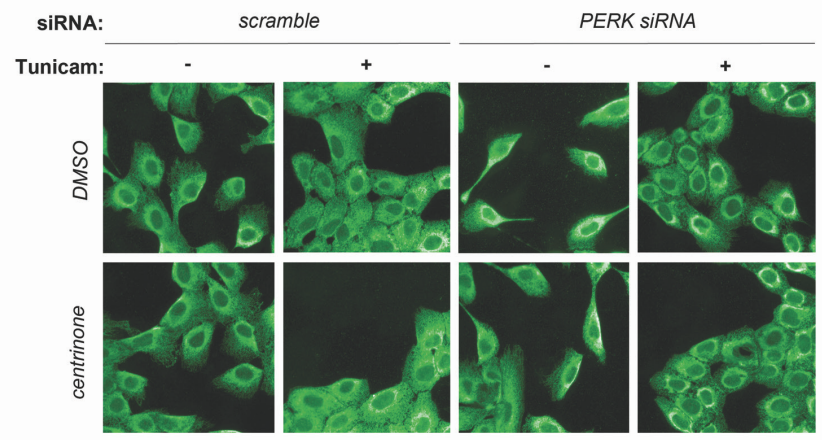

1097 Supplementary Figure 5.- (A) Image-based puromycylation assay across

1098 indicated conditions (4 biological replicates, approx. 2000 cells each). Data 1099 are expressed as normalized to control untreated condition. (B-C) ER 1100 remodeling assay (immunofluorescence images: calreticulin) across indicated 1101 conditions (4 biological replicates, approx. 2000 cells each). Statistical 1102 significance across assays was assessed by paired t-Student test; *: $p<0.05$; $1103{ }^{* *}: p<0.01 ;{ }^{* * *}: p<0.005$. n.s.: $p>0.05$ 

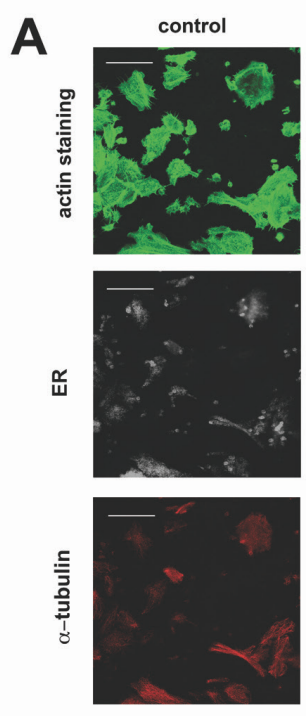

B
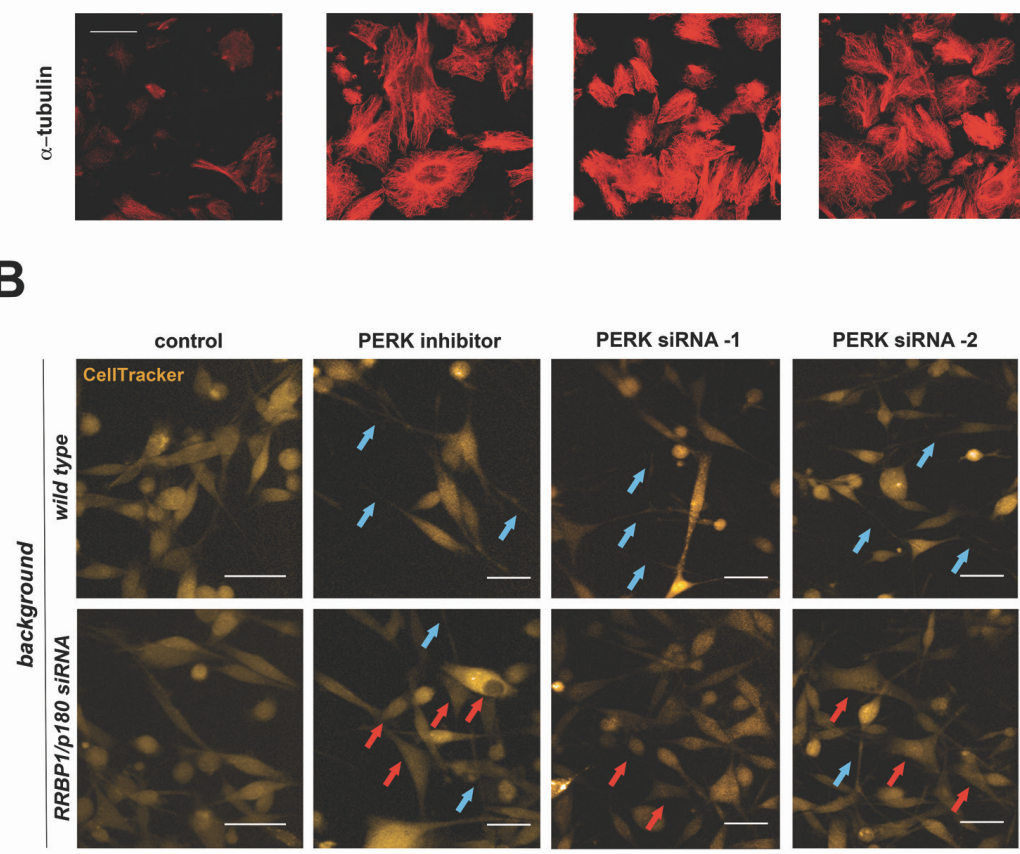
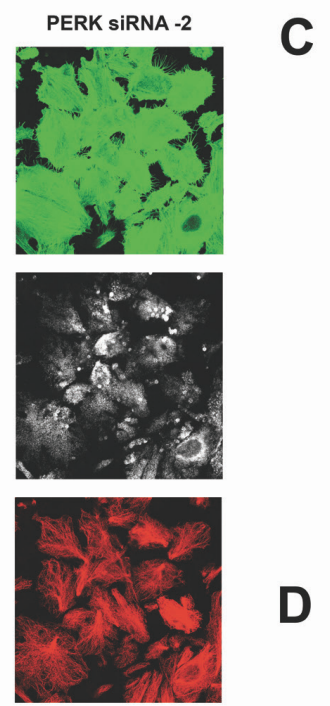

MCF10A vs Fv2E cells - total \% of migrating cells

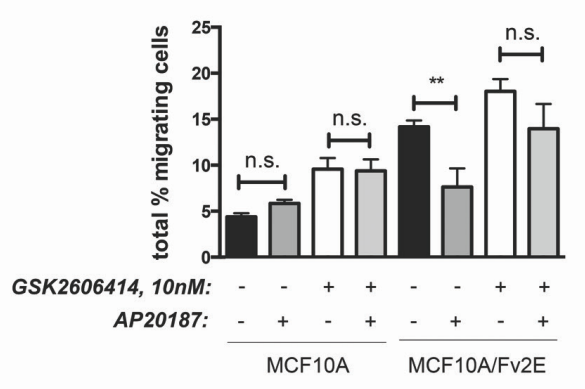

1106 Supplementary Figure 6.- (A) Control Transwell ${ }^{\mathrm{TM}}$ protrusion formation

1107 assay in MCF10A cells across indicated conditions and labels. PERK

1108 inhibitor: GSK2606414, 40nM (B) Cells subjected to indicated siRNA

1109 treatments were plated on soft collagen matrices, counterstained with

1110 CellTracker Orange, and imaged by confocal microscopy. Examples of cells

1111 with long protrusions (blue arrows), as opposed to cells with no apparent

1112 elongated protrusions (red arrows), are indicated. (C, D) Cell migration assays

1113 across indicated conditions and MCF10A clones, related to experiments

1114 shown in Fig. 7F. Data were derived from 6 biological replicates. Statistical

1115 significance across assays was assessed by paired t-Student test; *: $p<0.05$;

$1116{ }^{* *}: p<0.01 ;{ }^{* * *}: p<0.005$. n.s.: $p>0.05$ 


\section{Supplementary table 1}

\begin{tabular}{|l|l|l|l|}
\hline \multicolumn{4}{|c|}{ PRIMARY ANTIBODIES } \\
\hline Antigen & Supplier & Reference Num. & Usage \\
\hline Calreticulin & Abcam & ab2907 & Immunofluorescence (1:2000) \\
\hline pSer51 elF2a & Enzo Biosciences & BML-SA405 & Western Blot (1:1000), IF (1:250) \\
\hline elF2a & Abgent & P05198 & Western Blot (1:1000) \\
\hline Puromycylated peptide & DSHB & PMY-2A4 & IF (1:2000) \\
\hline HA tag & Roche & 11867423001 & Western Blot (1:1000) \\
\hline$\alpha$-tubulin & Sigma & T9026 & IF (1:2000) \\
\hline CAMSAP2 & ThermoScientific & PA5-99978 & Western Blot (1:1000) \\
\hline RRBP1 & ThermoScientific & MA5-18302 & IF (1:250) \\
\hline ATF4 & Santa Cruz & Sc-390063 & Western Blot (1:1000) \\
\hline
\end{tabular}

\begin{tabular}{|l|l|l|}
\hline \multicolumn{3}{|c|}{ siRNA/esiRNA } \\
\hline Target & Supplier & Reference \\
\hline PERK & Dharmacon & $\mathrm{M}-004883-03$ \\
\hline PERK & Dharmacon & $\mathrm{J}-004883-09$ \\
\hline PERK & Dharmacon & $\mathrm{J}-004883-10$ \\
\hline PERK & Dharmacon & $\mathrm{J}-004883-11$ \\
\hline PERK & Dharmacon & $\mathrm{J}-004883-12$ \\
\hline PERK & Dharmacon & $\mathrm{L}-004883-00$ \\
\hline PERK & Sigma & EHU030881 \\
\hline IRE1 & Dharmacon & $\mathrm{M}-004951-02$ \\
\hline ATF6 & Dharmacon & $\mathrm{M}-009917-01$ \\
\hline ATL1 & Dharmacon & $\mathrm{M}-010946-02$ \\
\hline RTN4 & Dharmacon & $\mathrm{M}-010721-00$ \\
\hline RTN1 & Dharmacon & $\mathrm{M}-014138-00$ \\
\hline ATL2 & Dharmacon & $\mathrm{M}-014047-00$ \\
\hline ATL3 & Dharmacon & $\mathrm{M}-010656-00$ \\
\hline SREBP1 & Dharmacon & $\mathrm{M}-006891-01$ \\
\hline CKAP4 & Dharmacon & $\mathrm{L}-012755-01$ \\
\hline RRBP1 & Dharmacon & $\mathrm{L}-011891-02$ \\
\hline ATF4 & Dharmacon & $\mathrm{L}-005125-00$ \\
\hline REEP4 & Dharmacon & $\mathrm{L}-016343-02$ \\
\hline REEP5 & Dharmacon & $\mathrm{L}-019467-01$ \\
\hline REEP6 & Dharmacon & $\mathrm{L}-015555-02$ \\
\hline CAMSAP2 & Dharmacon & $\mathrm{L}-022091-00$ \\
\hline CAMSAP2 & Sigma & EHU134911 \\
\hline
\end{tabular}

